\title{
Stellar activity analysis of Barnard's Star: very slow rotation and evidence for long-term activity cycle
}

B. Toledo-Padrón, ${ }^{1,2 \star ~ J . ~ I . ~ G o n z a ́ l e z ~ H e r n a ́ n d e z, ~}{ }^{1,2 \star}$ C. Rodríguez-López, ${ }^{3}$

A. Suárez Mascareño, ${ }^{4 \star}$ R. Rebolo, ${ }^{1,2,5}$ R. P. Butler, ${ }^{6}$ I. Ribas, ${ }^{7,8}$

G. Anglada-Escudé ${ }^{\oplus},{ }^{3,9}$ E. N. Johnson, ${ }^{10}$ A. Reiners, ${ }^{10}$ J. A. Caballero, ${ }^{11}$

A. Quirrenbach, ${ }^{12}$ P. J. Amado, ${ }^{3}$ V. J. S. Béjar, ${ }^{1,2}$ J. C. Morales, ${ }^{7,8}$ M. Perger, ${ }^{7,8}$

S. V. Jeffers, ${ }^{10}$ S. Vogt, ${ }^{13}$ J. Teske,${ }^{6}$ S. Shectman,,${ }^{14}$ J. Crane,${ }^{14}$ M. Díaz,${ }^{14,15}$

P. Arriagada, ${ }^{6}$ B. Holden, ${ }^{13}$ J. Burt, ${ }^{16}$ E. Rodríguez, ${ }^{3}$ E. Herrero, ${ }^{7,8}$ F. Murgas,,${ }^{1,2}$

E. Pallé, ${ }^{1,2}$ N. Morales,${ }^{3}$ M. J. López-González, ${ }^{3}$ E. Díez Alonso, ${ }^{17}$ M. Tuomi, ${ }^{18}$

M. Kiraga, ${ }^{19}$ S. G. Engle, ${ }^{20}$ E. F. Guinan, ${ }^{20}$ J. B. P. Strachan,,${ }^{9}$ F. J. Aceituno, ${ }^{3}$

J. Aceituno, ${ }^{21}$ V. M. Casanova, ${ }^{3}$ S. Martín-Ruiz, ${ }^{3}$ D. Montes ${ }^{\oplus},{ }^{22}$ J. L. Ortiz, ${ }^{3}$ A. Sota, ${ }^{3}$

J. Briol, ${ }^{23}$ L. Barbieri, ${ }^{23}$ I. Cervini, ${ }^{23}$ M. Deldem, ${ }^{23}$ F. Dubois, ${ }^{23,24}$ F. -J. Hambsch, ${ }^{23,25}$

B. Harris, ${ }^{23}$ C. Kotnik, ${ }^{23}$ L. Logie, ${ }^{23,24}$ J. Lopez, ${ }^{23}$ M. McNeely, ${ }^{23}$ Y. Ogmen, ${ }^{23}$

L. Pérez, ${ }^{23}$ S. Rau, ${ }^{23,24}$ D. Rodríguez, ${ }^{23}$ F. S. Urquijo ${ }^{23}$ and S. Vanaverbeke ${ }^{23,24}$

Affiliations are listed at the end of the paper

Accepted 2019 July 15. Received 2019 July 13; in original form 2018 December 18

\begin{abstract}
The search for Earth-like planets around late-type stars using ultrastable spectrographs requires a very precise characterization of the stellar activity and the magnetic cycle of the star, since these phenomena induce radial velocity (RV) signals that can be misinterpreted as planetary signals. Among the nearby stars, we have selected Barnard's Star (Gl 699) to carry out a characterization of these phenomena using a set of spectroscopic data that covers about $14.5 \mathrm{yr}$ and comes from seven different spectrographs: HARPS, HARPS-N, CARMENES, HIRES, UVES, APF, and PFS; and a set of photometric data that covers about $15.1 \mathrm{yr}$ and comes from four different photometric sources: ASAS, FCAPT-RCT, AAVSO, and SNO. We have measured different chromospheric activity indicators ( $\mathrm{H} \alpha, \mathrm{Ca}$ II $\mathrm{HK}$, and $\mathrm{Na}$ I D), as well as the full width at half-maximum (FWHM), of the cross-correlation function computed for a sub-set of the spectroscopic data. The analysis of generalized Lomb-Scargle periodograms of the time series of different activity indicators reveals that the rotation period of the star is $145 \pm 15 \mathrm{~d}$, consistent with the expected rotation period according to the low activity level of the star and previous claims. The upper limit of the predicted activity-induced RV signal corresponding to this rotation period is about $1 \mathrm{~m} \mathrm{~s}^{-1}$. We also find evidence of a long-term cycle of $10 \pm 2 \mathrm{yr}$ that is consistent with previous estimates of magnetic cycles from photometric time series in other M stars of similar activity levels. The available photometric data of the star also support the detection of both the long-term and the rotation signals.
\end{abstract}

Key words: stars: activity-stars: individual: Barnard's star (Gl 699)-stars: rotation.

\section{INTRODUCTION}

Since the discovery of the first extrasolar planet in 1992 (Wolszczan \& Frail 1992) and the detection of the first exoplanet orbiting a solar-type star (Mayor \& Queloz 1995), 3884 exoplanets have been 
detected using different techniques. ${ }^{1}$ One of the most commonly used methods is the radial velocity (RV) technique, which has been applied to find 773 extrasolar planets around 576 stars. The majority of these stars are $\mathrm{G}$ or $\mathrm{K}$ type (with a percentage of 42 percent and 33 per cent, respectively), and only 49 of them are M-dwarfs ( 8 per cent of the total sample), the first one detected in 2001 around GJ 876 (Marcy et al. 2001). The search for Earth-like planets around these $\mathrm{M}$ type stars takes advantage of having greater amplitudes in the RV planetary signals due to the low mass of their parent star. Also, this type of stars is the most common stellar type in the Milky Way (Chabrier \& Baraffe 2000). However, stellar activity in M-dwarfs can produce signals with periods commensurate with the 'habitable zones' around these stars (Newton et al. 2016b; Vanderburg et al. 2016), where liquid water could potentially exist on the surface of a planet. Distinguishing whether signals arise from orbiting planets or stellar activity can be challenging. The signals produced by stellar activity are on the time-scale of the rotation period of the star, but we also have to take care of the long-period signals associated with Doppler shifts caused by the magnetic cycle of the star (Dravins 1985; Campbell, Walker \& Yang 1988) already reported around M stars (Gomes da Silva et al. 2012; Robertson et al. 2013; Díez-Alonso et al. 2019). Photon noise of the measurements is a key selection criteria of stellar samples in RV search programs. The high SNR of nearby stars makes them very interesting targets for low-mass exoplanets searches. Among the nearby stars, we have selected the closest single M-dwarf to the Solar system: Barnard's Star (Gl 699).

Barnard's Star is well known for being the second closest stellar system to the Sun. Located at a distance of 1.8 parsecs (Brown et al. 2018), and with an age between 7 and 10 Gyr (Ribas et al. 2018), Gl 699 is the star with the highest proper motion known to date (Barnard 1916), which causes Doppler shifts due to secular acceleration (Stumpff 1985; Kürster et al. 2003) that needs to be taken into account for exoplanet searches through RV. It also presents a low X-ray luminosity, which indicates a low level of current magnetic activity (Vaiana et al. 1981; Hünsch et al. 1999; Marino, Micela \& Peres 2000). This reduces the effects of spots and plages in the spectral line profiles (Lovis et al. 2011). The most important properties of this star are shown in Table 1.

Previous work carried out on this star (Suárez Mascareño et al. 2015; Astudillo-Defru et al. 2017) has revealed a low level of chromospheric emission $\left(\log R_{\mathrm{HK}}^{\prime}=-5.86\right.$ and $\log R_{\mathrm{HK}}^{\prime}=-5.69$, respectively), which is usually related to slow rotators. Using these two values in the relation between the rotation period and the activity level of the star predicted by Suárez Mascareño et al. (2016) gives an expected rotation period of 152 and $112 \mathrm{~d}$, respectively. This range is in good agreement with the previous value of $130 \mathrm{~d}$ given by Benedict et al. (1998) through a photometric study using the Hubble Space Telescope. Also Suárez Mascareño et al. (2015) reported a $148.6 \mathrm{~d}$ rotation period obtained from a time-series analysis of spectroscopic indexes using High Accuracy Radial velocity Planet Searcher (HARPS) data.

Recently, Ribas et al. (2018) reported the discovery of a superEarth like planet orbiting Barnard's Star at an orbital period of $233 \mathrm{~d}$ with a minimum mass of 3.3 Earth masses. We will focus on the stellar activity and magnetic cycle characterization through a multispectrograph analysis of several activity indexes, complemented by a multi-instrumental analysis of photometric time series, which leads to detect and increase the precision in the rotation

${ }^{1}$ source: http://www.exoplanet.eu
Table 1. Stellar properties of Barnard's Star.

\begin{tabular}{lcc}
\hline Parameter & Gl 699 & Ref. \\
\hline RA $(\mathrm{J} 2000)$ & $17: 57: 48.50$ & {$[1]$} \\
Dec. $(\mathrm{J} 2000)$ & $+04: 41: 36.11$ & {$[1]$} \\
$\mu_{\alpha} \cos \delta\left(\mathrm{mas} \mathrm{yr}^{-1}\right)$ & $-802.8 \pm 0.6$ & {$[1]$} \\
$\mu_{\delta}\left(\mathrm{mas} \mathrm{yr}^{-1}\right)$ & $+10362.5 \pm 0.4$ & {$[1]$} \\
Distance $(\mathrm{pc})$ & $1.8266 \pm 0.0001$ & {$[1]$} \\
$m_{\mathrm{B}}$ & 11.24 & {$[2]$} \\
$m_{\mathrm{V}}$ & 9.51 & {$[2]$} \\
Spectral type & $\mathrm{M} 3.5 \mathrm{~V}$ & {$[3]$} \\
$T_{\text {eff }}(\mathrm{K})$ & $3278 \pm 51$ & {$[4]$} \\
{$[\mathrm{Fe} / \mathrm{H}](\mathrm{dex})$} & $-0.12 \pm 0.16$ & {$[4]$} \\
$M_{\star}\left(\mathrm{M}_{\odot}\right)$ & $0.163 \pm 0.022$ & {$[5]$} \\
$R_{\star}\left(\mathrm{R}_{\odot}\right)$ & $0.178 \pm 0.011$ & {$[5]$} \\
$L_{\star}\left(\mathrm{L}_{\odot}\right)$ & $0.00329 \pm 0.00019$ & {$[5]$} \\
$\log g(\mathrm{cgs})$ & $5.10 \pm 0.07$ & {$[4]$} \\
$\log \left(L_{\mathrm{X}} / L_{\text {bol }}\right)$ & -5.4 & {$[6]$} \\
$v \sin i\left(\mathrm{~km} \mathrm{~s}^{-1}\right)$ & $<3$ & {$[4]$} \\
$a_{\text {sec }}\left(\mathrm{m} \mathrm{s}^{-1} \mathrm{yr}^{-1}\right)$ & $5.15 \pm 0.89$ & {$[7]$} \\
$\log \left(R_{10}^{\prime}\left(R_{\mathrm{HK}}\right)\right.$ & $-5.82 \pm 0.08$ & {$[8]$} \\
$P_{\text {rot }}(\mathrm{d})$ & $145 \pm 15$ & {$[8]$} \\
$\operatorname{Long}-\mathrm{term}$ activity cycle $(\mathrm{d})$ & $3800 \pm 600$ & {$[8]$} \\
\hline
\end{tabular}

Note. References: [1] Brown et al. (2018); [2] Koen et al. (2010); [3] AlonsoFloriano et al. (2015); [4] Passegger et al. (2018); [5] Ribas et al. (2018); [6] Kiraga \& Stepien (2007); [7] Kürster et al. (2003); [8] This work.

Table 2. Properties of all the spectrographs used in this work.

\begin{tabular}{lccc}
\hline Spectrograph & $R$ & $\Delta \lambda(\AA)$ & $N_{\text {spec }}$ \\
\hline HARPS & 115000 & $3780-6910$ & 317 \\
HARPS-N & 115000 & $3830-6930$ & 74 \\
CARMENES & 90000 & $5200-17100$ & 192 \\
HIRES & 67000 & $3700-10000$ & 179 \\
UVES & 130000 & $3000-11000$ & 57 \\
PFS & 80000 & $3880-6680$ & 43 \\
APF & 100000 & $3740-9700$ & 95 \\
\hline
\end{tabular}

Note. Columns: Name of the spectrograph, resolution, spectral range, and number of spectra used in this work. The observation programs are listed in the acknowledgments.

period value and also to detect a long-term activity cycle in the star.

In Section 2, we describe the whole data set, both the spectroscopy and photometry used in this work. In Section 3, we describe the methodology used in the analysis of each stellar activity indicator. In Section 4, we show this analysis and the results obtained for each activity indicator. In Section 5, we discuss the results, and we provide the conclusions of this study in Section 6.

\section{DATA}

\subsection{Spectroscopic data set}

For this work we have used spectra taken with seven different spectrographs, whose main properties are shown in Table 2.

HARPS is a fiber-fed Echelle spectrograph installed in 2003 at the $3.6 \mathrm{~m}$ telescope of La Silla Observatory, Chile (Mayor et al. 2003). The spectra used in this work were collected between 2007 April (BJD = 2454194.9) and 2017 September (BJD = 2458027.5) with an exposure time of $900 \mathrm{~s}$. In the treatment of these data, we performed a separate analysis of the spectra taken before and after 2015 May. This is because on that date the vacuum vessel 
that contains the spectrograph was opened to upgrade the fibre link (Lo Curto et al. 2015), creating a discontinuity in the RV and index values, which necessitates of calculating an offset between the 'Pre-2015' and 'Post-2015' values. The instrument used for the wavelength calibration was a Thorium-Argon lamp (Lovis \& Pepe 2007), which provides a large number of spectral lines distributed in the visible spectral range of HARPS. For the most recent data, we used an ultrastable Fabry-Perot interferometer (Wildi et al. 2010), which provides the best short-term accuracy in RV determination from the instrument.

HARPS-N is the northern counterpart of HARPS. This instrument was installed in 2012 at the $3.6 \mathrm{~m}$ Telescopio Nazionale Galileo (TNG) in Roque de los Muchachos Observatory, Spain (Cosentino et al. 2012). It has the same resolution as HARPS, similar wavelength coverage and is also contained in a vacuum vessel to minimize the temperature and pressure variations that may cause spectral drifts. The spectra used were taken between 2014 July (BJD $=2456841.5)$ and 2017 October (BJD = 2458038.4) with the same exposure time used in HARPS. The wavelength calibration was also done using a Th-Ar lamp.

CARMENES (Calar Alto high-Resolution search for M dwarfs with Exoearths with Near-infrared and optical Echelle Spectrographs) is a second generation Echelle spectrograph installed in 2015 at the $3.5 \mathrm{~m}$ telescope in Calar Alto Observatory, Spain (Quirrenbach et al. 2018). This instrument has two different channels that work simultaneously in the visible and near-infrared, and it is mainly focused on searching for low-mass planets in the habitable zones of late-type stars. The spectra used were acquired between 2016 February (BJD = 2457422.7) and 2017 October (BJD = 2458032.3), and we only use the visible channel. The calibration method is similar to the one used in HARPS, along with simultaneous Fabry-Perot exposures and a daily calibration using Th-Ne, U-Ar, and U-Ne lamps (Quirrenbach et al. 2018).

Ultraviolet-Visual Echelle Spectrograph (UVES) is a highresolution optical spectrograph installed in 2000 at the $8.2 \mathrm{~m}$ VLT in Paranal Observatory, Chile (Dekker et al. 2000). The spectra used in this work were taken between 2003 April $(\mathrm{BJD}=2452743.4)$ and 2005 October $(\mathrm{BJD}=2453658.0$ ). These spectra were acquired using an image slicer, with an effective slit width of 0.3 arcsec that gives a resolution of $\sim 130000$. UVES data are calibrated using the standard Th-Ar lamp, and in addition, accurate RVs are extracted thanks to an additional calibration based on an Iodine Cell, which provides many absorption lines on top of the target spectrum in some spectral regions. This makes some parts of the spectra not useful to measure for instance certain chromospheric indexes like the $\mathrm{Na}$ I D.

HIgh-Resolution Echelle Spectrometer (HIRES) is a firstgeneration Echelle spectrograph installed in 1996 at the $10 \mathrm{~m}$ Keck telescope in Mauna Kea Observatory, USA (Vogt et al. 1994). The spectra used were collected between 2004 August (BJD $=2453237.9$ ) and 2014 September (BJD = 2456908.7). The wavelength calibration was done in a similar way as for the UVES spectra, i.e. inserting the Iodine Cell in the light beam with the aim of improving the RV precision.

Carnegie Planet Finder Spectrograph (PFS) is a high-resolution optical Echelle spectrograph installed in 2009 at the $6.5 \mathrm{~m}$ Magellan II telescope in Las Campanas Observatory, Chile (Crane et al. 2010). The spectra we use were taken between 2011 August (BJD = 2455791.6) and 2016 August (BJD = 2457615.6). The wavelength calibration method is the same as the one used in UVES and HIRES.

The Automated Planet Finder (APF) consists of a $2.4 \mathrm{~m}$ telescope with the Levy Spectrometer commissioned in 2013 at the
Lick Observatory, USA (Vogt et al. 2014). The spectra we use were acquired between 2013 July (BJD = 2456504.7) and 2016 March (BJD = 2457478.0). This instrument has a similar optical configuration to PFS, and also uses an Iodine Cell to make the wavelength calibration.

\subsection{Photometric data set}

For the photometric analysis, we relied on data taken with four different sources. Archival publicly available data come from the All Sky Automated Survey (ASAS) survey, which has a time base of several years.

ASAS consists of two automated observing stations at Las Campanas Observatory, Chile (ASAS-S or ASAS-3), and Haleakalā Observatory, USA (ASAS-N or ASAS-3N) (Pojmański et al. 1997). These two stations observe simultaneously in the $V$ and $I$ photometric bands with an average accuracy of $\sim 0.05$ mag per exposure. They are complemented with the ASAS-SN (All-Sky Automated Survey for Supernovae) project (Shappee et al. 2014), which consists of 20 telescopes distributed around the globe that are automatically surveying the entire available sky every night down to $V \sim 17$ mag. Data from the ASAS-S and ASAS-SN were retrieved from its public data base ${ }^{2}$ while data from ASAS-N were supplied by M. Kiraga (private communication), as they have not yet been made public. We thus collect 836 epochs (measurements averaged to one per night) from this survey (coming from ASAS-N, ASAS-S, and ASAS-SN) that were taken between 2002 September $(\mathrm{BJD}=$ 2452524.6) and 2017 October (BJD $=2458032.7$ ).

Our own data comprise the second longest data set of all, after ASAS, coming from the Four College Automated Photoelectric Telescope (FCAPT) and the Robotically Controlled Telescope (RCT), with a time-span covering $14.5 \mathrm{yr}$.

The FCAPT is a $0.75 \mathrm{~m}$ automated telescope installed at the Fairborn Observatory (USA) that provides differential Strömgren $u v b y$, Johnson $B V$, and Cousins RI photometry of a wide variety of stars (Adelman et al. 2001). RCT is a $1.3 \mathrm{~m}$ telescope installed at the Kitt Peak National Observatory (USA) that includes an UBVRI broad-band filter set and is focused on observing faint objects such as brown dwarfs (Gelderman 2001). The combined data set from these two instruments is composed by 348 epochs, acquired in the $V$ Johnson filter, which were taken between 2003 May (BJD = 2452764.0) and 2017 June (BJD = 2457922.8).

In addition, we orchestrated a joint photometric follow-up campaign for Barnard's Star, quasi-simultaneous to its Doppler observations acquired as part of the Red Dots 2017 (RD2017) campaign, ${ }^{3}$ designed to search for planet signatures around our closest $\mathrm{M}$ dwarf neighbours. The participating observatories were as follows:

The Sierra Nevada Observatory (SNO, Spain), whose data come from the $0.9 \mathrm{~m}$ telescope (T90) that is equipped with a CCD camera VersArray $2 \mathrm{~K} \times 2 \mathrm{~K}$ with a $13.2 \times 13.2 \mathrm{arcmin}^{2}$ field of view. We work with 69 epochs from this telescope that were taken since 2017 May (BJD = 2457887.6) until 2017 October (BJD = 2458042.3), quasi-simultaneous to the RD2017 campaign. We collected about 30 measurements per night in each in $B, V$, and $R$ Johnson filters, accounting for a total of about 2000 observations in each filter.

The Montsec Astronomical Observatory (OAdM, Spain), whose data come from the Joan Oró robotic telescope (TJO) that

\footnotetext{
${ }^{2} \mathrm{http} / / /$ www.astrouw.edu.pl/asas/

${ }^{3}$ https://reddots.space/
} 
is equipped with a CCD Andor DW936N-BV camera with a $12.3 \times 12.3 \mathrm{arcmin}^{2}$ field of view. We work with 72 epochs from this telescope that were taken since 2017 June (BJD $=2457920.5$ ) until 2017 October (BJD = 2458050.3), quasi-simultaneous to the RD2017 campaign. A minimum of 5 measurements was done per night, to finally obtain a total of about 700 images in two filters $(R$ and $I$ ). As the majority of photometric data from other instruments were acquired in the $V$ filter, we do not include the OAdM data set in the final analysis.

Following the outreach spirit of the Pale Red Dot campaign (Anglada-Escudé et al. 2016), our desire was that the RD2017 campaign involved as many members of the public as possible. Thus, in addition to the set-up of the RD2017 website $^{3}$ and social media for the campaign, we requested support from the AAVSO (American Association of Variable Stars Observers) and issued an AAVSO alert with a call for photometric follow-up from observers. The answer was enthusiastic, with more than 8000 measurements in the $B V R I$ and $\mathrm{H} \alpha$ filters for Barnard's Star uploaded to the AAVSO data base from 14 observers in eight countries (see Table A3). About 75 per cent of the observations/acquired exposures (or half of the data sets) had great quality and could be included in the analysis, covering a time-span of $120 \mathrm{~d}$ with 6310 measurements in 148 epochs, as measurements from different observers were not consolidated into nightly binned epochs.

We also analysed data from the Las Cumbres Observatory network (LCO) and the ASH2 $0.40 \mathrm{~m}$ telescope at SPACEOBS (San Pedro de Atacama Celestial Explorations Observatory) observatory (Chile), the latter being operated by the Instituto de Astrofísica de Andalucía (IAA).

In the case of LCO, data were obtained in the $B$ and $V$ Johnson and $r^{\prime}$ and $i$ ' Sloan filters. Unfortunately, data in the $B, r$ ', and $i$ ' filters could not be used due to instrumental issues. The data in the $V$ filter were not included either in the final combined data set due to their high dispersion both intra and night to night, as reflected in the high mean error and root mean square (rms), of 16.0 and $30.5 \mathrm{mmag}$, respectively. The scattering was very high in comparison to the other observatories simultaneously acquiring data in the RD2017 campaign.

In the case of SPACEOBS, observations were acquired in three narrow-band filters with an FWHM of $12 \mathrm{~nm}$, centred on the O III (501 nm), S II (672 nm), and $\mathrm{H} \alpha(656 \mathrm{~nm})$ lines, with mean errors in the range of 14-24 mmag, larger than in most data sets. This is most likely attributed to the narrow filters and faint comparison stars. The night-to-night stability, shown by the rms, is low, with values ranging from 7 to $13 \mathrm{mmag}$, depending on the filter. The narrowband lines were useful to monitor any possible activity bursts, such as flares, but were not included in the final combined data set due to the short time base and larger scatter compared to the other RD2017 observatories simultaneously acquiring data.

Finally, we also analyse publicly available data from the MEarth survey, which consists of two arrays of robotically controlled telescopes located at the Fred Lawrence Whipple Observatory (USA) and Cerro Tololo Inter-American Observatory (Chile) (Berta et al. 2012). Each array consists of eight identical telescopes with a $0.4 \mathrm{~m}$ primary mirror that focuses the light on to a high-grade CCD camera with a broad RG715 nm filter. We work with 161 epochs from this survey that were taken since 2013 February (BJD $=2454876.0$ ) until 2015 October (BJD $=2457323.6$ ). The large mean error in this data set indicates that the measurements had a large intranight scatter, but once consolidated into nightly averages, the scatter of the whole run decreased to $6.5 \mathrm{mmag}$, indicating that there were not large differences from night-to-night observations.
Table 3. Properties of the photometric data

\begin{tabular}{|c|c|c|c|c|}
\hline $\begin{array}{l}\text { Observatory/ } \\
\text { Survey/ } \\
\text { Telescope }\end{array}$ & $\begin{array}{l}\text { Aperture } \\
\text { (m) }\end{array}$ & Filter & $\begin{array}{l}\text { Error } \\
(\mathrm{mmag})\end{array}$ & $\begin{array}{c}\mathrm{rms} \\
(\mathrm{mmag})\end{array}$ \\
\hline ASAS-3 & 0.07 & $V$ & 10.3 & 17.0 \\
\hline ASAS-3N & 0.10 & V & 13.0 & 16.1 \\
\hline ASAS-SN & 0.14 & $V$ & 5.2 & 8.3 \\
\hline CombinedASAS & $0.07,0.10,0.14$ & $V$ & 10.4 & 16.8 \\
\hline $\begin{array}{l}\text { FCAPT and } \\
\text { RCT }\end{array}$ & $0.80,1.30$ & $V$ & 4.9 & 11.2 \\
\hline MEarth & 0.40 & RG715 & 16.5 & 6.5 \\
\hline \multirow[t]{3}{*}{ SNO } & 0.90 & $B$ & 4.5 & 5.4 \\
\hline & & $V$ & 4.4 & 6.4 \\
\hline & & $R$ & 5.8 & 5.3 \\
\hline \multirow[t]{2}{*}{ OAdM } & 0.80 & $R$ & 7.2 & 9.6 \\
\hline & & $I$ & 8.4 & 8.8 \\
\hline AAVSO & Range & V & 15.1 & 8.9 \\
\hline \multirow[t]{3}{*}{ LCO } & 0.40 & V & 16.0 & 30.5 \\
\hline & & $r^{\prime}$ & 31.1 & 45.2 \\
\hline & & $i^{\prime}$ & 91.4 & 75.6 \\
\hline \multirow[t]{3}{*}{ ASH2 } & 0.40 & [O III] & 14.1 & 7.1 \\
\hline & & $\mathrm{H} \alpha$ & 23.9 & 12.5 \\
\hline & & [S II] & 16.8 & 9.5 \\
\hline
\end{tabular}

Note. Columns: Observatory, survey, or telescope; telescope aperture; filters; mean error of the nights and rms of the run (see the text for details).

We did not combine this data set with the rest of the time series because it was taken with a filter that did not match the $V$ Johnson filter used in the other data sets.

The properties of all of these photometric sources are shown in Table 3, including the mean error of the averaged nights, which indicates the scatter of the measurements within the night, giving an idea of the quality of the nights; and the rms of the run, which gives a measure of the night-to-night stability. We mark in boldface the selected data sets that we used for the analysis presented in this paper (using the $V$-filter time series in each case).

All photometric data (except for ASAS and MEarth) were reduced with standard procedures including bias and/or dark subtraction and flat-field correction. Several apertures were tried to extract the best aperture photometry that maximized the signalto-noise ratio (SNR). Differential magnitudes were obtained with respect to nearby comparison stars that had previously been checked for stability and, in the case of observations taking place during the RD2017 campaign, agreed upon, so that the different photometric data sets were as uniform and comparable as possible.

\section{METHOD}

\subsection{Determination of stellar activity indicators}

In order to measure activity indices, we first correct all the spectra for the blaze function. In the case of HARPS and HARPS-N we use a specific blaze spectrum given by their respective pipelines, and for the other spectrographs we fit a second-order polynomial to each order to create an artificial blaze spectrum.

Next, we correct for the pixel size variability in wavelength, which requires to re-binning the spectra to obtain a constant step 


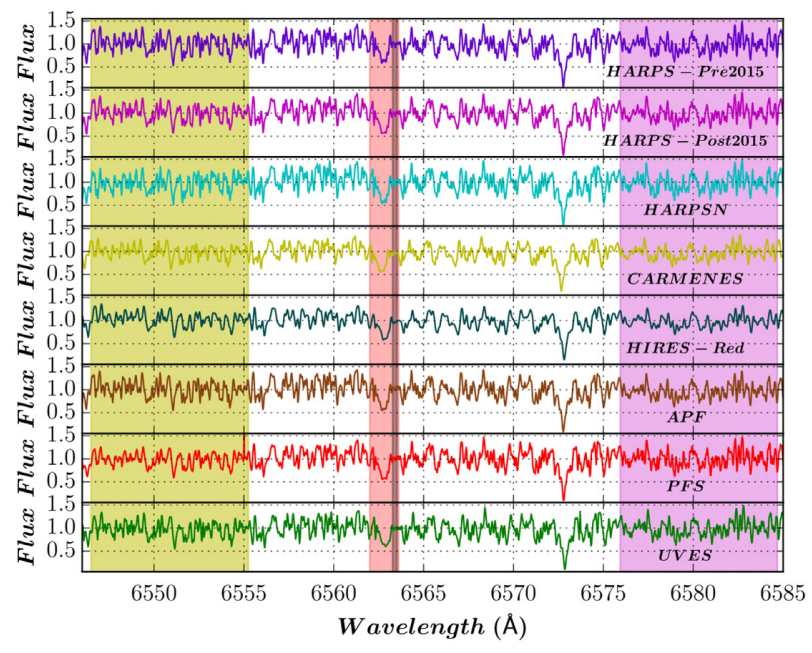

Figure 1. Normalized one-dimensional spectra taken with seven spectrographs. The $\mathrm{H} \alpha$ band is marked in pink, the continuum passbands are marked in yellow and violet and the continuum region used to calculate the index error is marked in grey.

in wavelength between pixels and also to correct accordingly the flux evaluated in the selected wavelength step $(0.01 \AA)$. Then we correct the wavelength for the barycentric velocity of the Earth and the RV of the star. Both velocities are available in the header of the HARPS, HARPS-N, and CARMENES spectra. In the case of HIRES, APF, PFS, and UVES, we calculate the barycentric velocity using the equatorial coordinates (RA and Dec.) and the Julian day (BJD), and we use a calculated value of $-110.25 \mathrm{~km} \mathrm{~s}^{-1}$ for the RV (obtained by averaging the HARPS and HARPS-N header values). To deal with the small wavelength shifts (few $\mathrm{m} \mathrm{s}^{-1}$ ) related to the use of a mean value for the RV instead of variable value over time, we correlate the spectra in these four spectrographs using the first spectrum of each spectrograph as reference. Finally, we build an average spectrum and use the individual spectra to calculate the weights of each Echelle order involved in the index. The weight of one order in a certain spectrum is calculated as the quotient between the normalized median of the flux of this order in the average spectrum and the selected spectrum.

Once all the spectra have passed through this process, we can measure the three activity indices. The first one is the $\mathrm{H} \alpha$ index, which we define as:

$\mathrm{H} \alpha=\frac{A}{L+R}$,

where $A$ is a rectangular passband centred at the core of the $\mathrm{H} \alpha$ line (6562.808 $\AA$ ) with a width of $1.6 \AA$, and $L$ and $R$ are the continuum bands centred at 6550.870 and $6580.310 \AA$, respectively, with a width of $8.75 \AA$ (Kürster et al. 2003; Gomes da Silva et al. 2011). Fig. 1 shows this spectral region for the seven spectrographs in which it is possible to measure this index.

The second one is similar to the S-index related to the Ca II $\mathrm{H}$ \& $\mathrm{K}$ lines (Noyes et al. 1984), that we call CaHK index and define as

$S=\frac{H+K}{R+V}$,

where $H$ and $K$ are triangular passbands for the core of the lines (centred at 3968.470 and $3933.664 \AA$ A, respectively) with an FWHM of $1.09 \AA$. In this work we have shifted the continuum filters of $R$ and $V$ from 4001.070 and $3901.070 \AA$, to 3976.5 and $3925.5 \AA$, respectively, and also modified the width of both filters from 20

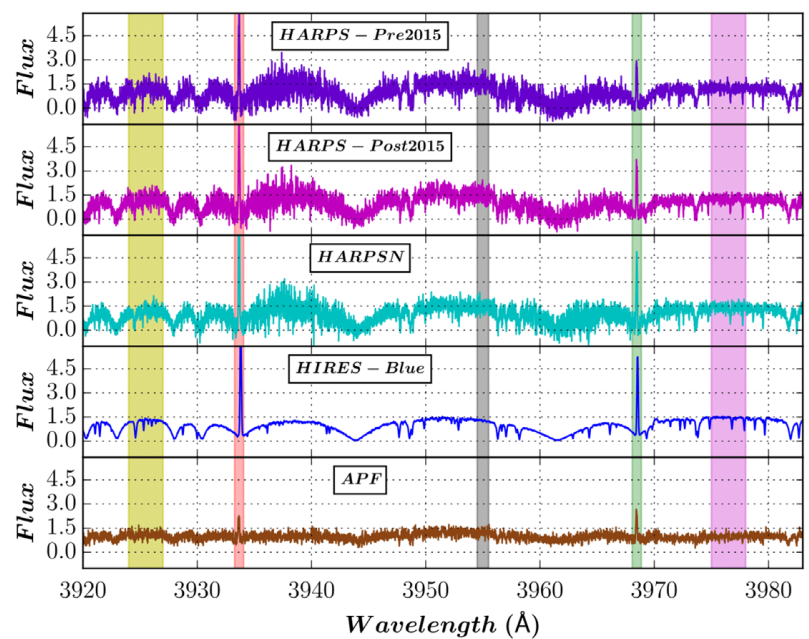

Figure 2. Normalized one-dimensional spectra taken with four spectrographs. The $\mathrm{Ca}$ II $\mathrm{H} \& \mathrm{~K}$ bands are marked in green and pink, respectively, the continuum passbands are marked in yellow and violet and the continuum region used for the index error is marked in grey.

to $3 \AA$, in order to use narrower spectral regions near the core of the lines located in the same Echelle orders as those lines. These continuum bands allow us to avoid the overlap between different Echelle orders in all of the spectrographs. Fig. 2 shows this spectral region for the four spectrographs in which it is possible to measure this index.

The last activity indicator is the Na I $D$ index (Díaz, Cincunegui \& Mauas 2007), which we define as

$N=\frac{D_{1}+D_{2}}{L+R}$,

where $D_{1}$ and $D_{2}$ are rectangular passbands for the core of the sodium doublet lines (centred at 5895.92 and $5889.95 \AA$, respectively) with a width of $1 \AA$. $L$ and $R$ are the continuum bands that are usually centred at 5805.0 and $6090.0 \AA$, with a width of 10 and $20 \AA$, respectively, but in this work we shifted them to 5881.5 and 5902.5 $\AA$. We also modified their widths to $12 \AA$ for the same reason as the one used for the CaHK index. Fig. 3 shows this spectral region for the five spectrographs in which it is possible to measure this index.

The uncertainties of the three indices were determined through error propagation (Taylor 1982), using the rms of the error region marked in grey in Figs $1-3$ as the error for the bands $A, L, R, H, K$, $V, D_{1}$, and $D_{2}$.

We also used the cross-correlation function (CCF) computed by the HARPS, HARPS-N, and CARMENES pipelines to estimate the FWHM as an additional activity indicator. We computed an average CCF using individual weights for each Echelle order as we did with the spectra (we build an average CCF and use the individual CCFs to calculate the weights of each Echelle order). Then we cut the $\mathrm{CCF}$ to a width of 25 pixels and used a Gaussian + second-order polynomial fit to obtain the FWHM. In Fig. 4 four CCF fits are shown, one per spectrograph, along with their residuals.

Once we had the measurements from all the spectra, we computed the weighted average per night, discarding those values that are beyond $3 \sigma$ from the median index in order to remove outliers. We also discarded values with errors beyond $3 \sigma$ from the median error. The outliers may be associated in some cases with flares, a phenomenon already detected in Barnard's Star (Paulson et al. 2006), though occurring rarely due to its advanced 


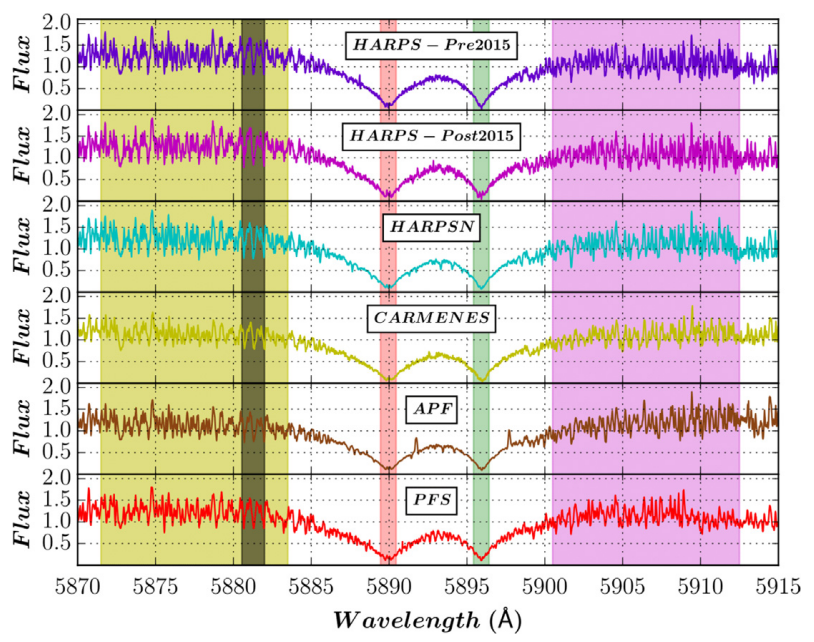

Figure 3. Normalized one-dimensional spectra taken with five spectrographs. The Na I $D_{1}$ and $D_{2}$ bands are marked in pink and green, respectively, the continuum passbands are marked in yellow and violet and the continuum region used for the index error is marked in grey.

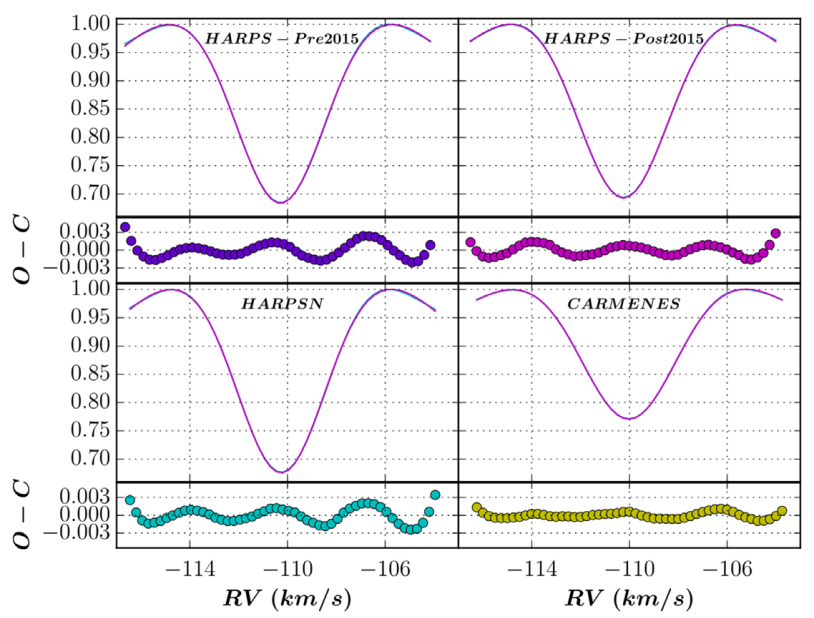

Figure 4. CCFs obtained for a single observation of three different spectrographs along with their respective residuals.

age. We also applied this treatment to the photometric data, in which we already had a set of magnitudes measured with different instruments. This process gave the final number of datapoints shown in Table 4 for the four spectroscopic indices and the photometric magnitudes.

The relative offsets between instruments were calculated for each index separately. We divided the spectroscopic data into two separate blocks according to their time-span in order to have enough overlapping observations: the first one included HIRES, HARPSPre2015, PFS, APF, and UVES; and the second one included HARPS-Post2015, HARPS-N, and CARMENES. We used time windows of $10 \mathrm{~d}$ for spectrographs of the same block, and $30 \mathrm{~d}$ for spectrographs of different blocks. We determined the difference between the values contained in these windows and averaged all of them to obtain the offset. For the photometric data, we only used one block of instruments due to the long-time coverage of surveys like ASAS. These offsets are shown in Table 5.

After applying these offsets, we apply another $3 \sigma$ clipping to the complete data set values and we get the time series (of each spectroscopic index and photometric magnitude) shown in Fig. 5.
Table 4. Numberof measurements used for every index after the selection criteria were applied.

\begin{tabular}{lccccc}
\hline Instrument & \multicolumn{5}{c}{$N_{\text {measurements }}$} \\
& $\mathrm{H} \alpha$ & $\mathrm{CaHK}$ & $\mathrm{NaD}$ & FWHM & $m_{\mathrm{V}}$ \\
\hline HARPS-Pre2015 & 109 & 110 & 114 & 115 & - \\
HARPS-Post2015 & 66 & 66 & 63 & 65 & - \\
HARPS-N & 40 & 39 & 40 & 40 & - \\
CARMENES & 182 & - & 164 & 173 & - \\
HIRES & 124 & 125 & - & - & - \\
APF & 44 & 45 & 42 & - & - \\
PFS & 33 & - & 30 & - & - \\
UVES & 21 & - & - & - & - \\
ASAS & - & - & - & - & 830 \\
FCAPT-RCT & - & - & - & - & 344 \\
AAVSO & - & - & - & - & 148 \\
SNO & - & - & - & - & 68 \\
Combined & 619 & 385 & 453 & 393 & 1390 \\
\hline
\end{tabular}

\subsection{Time-series analysis}

We carry out a time-series analysis of the three spectroscopic activity indicators, the CCF FWHM and the $V$-band photometry using the Lomb-Scargle periodogram (Lomb 1976) in its generalized form (Zechmeister \& Kürster 2009), in which each value has an independent error. We also analyse the chromatic index (CRX) given only in the CARMENES data. Each point of the periodogram is calculated as

$z(\omega)=\frac{N-3}{2} \times p(\omega)=\frac{N-3}{2} \times \frac{\chi_{o}^{2}-\chi^{2}(\omega)}{\chi_{o}^{2}}$,

where $N$ are the degrees of freedom and $\chi^{2}$ is the squared difference between the data and the model for a certain frequency $\omega$, calculated as

$\chi^{2}=\sum_{i=1}^{N} \frac{\left[y_{i}-y\left(t_{i}\right)\right]^{2}}{\sigma_{i}^{2}}$.

The false alarm probability (FAP) (Horne \& Baliunas 1986) associated with every point in the periodogram is calculated with the following expression (Cumming 2004):

$\mathrm{FAP}=1-\left[1-P\left(z>z_{o}\right)\right]^{M}=1-\left[1-e^{-z_{o}}\right]^{M}$,

where $z$ is the real power of a point in the periodogram, $z_{o}$ is the measured power, $M$ is the number of independent frequencies used in the periodogram, and $P\left(z>z_{o}\right)$ measures the probability of $z$ being greater than $z_{o}$. Using equation (6) we established a first approximation of the 10 per cent, 1 per cent, and 0.1 per cent levels of FAP for the periodograms. To obtain more precise values of these levels we applied a bootstrapping method (Endl et al. 2001). This method involves re-arranging the time order of the indices values 10000 times, searching for the period with the highest significance in every iteration to determine which values are obtained 10 per cent, 1 per cent, and 0.1 per cent of the times.

In Fig. 6 we show the periodograms for the four spectroscopic indices and the $V$ magnitude using the time series from Fig. 5 (which includes all the instruments used with their respective offsets, except in the case of the photometric time series, where we did not use the FCAPT-RCT data set for reasons that will be discussed later) with the FAP levels from bootstrapping. Signals with an FAP lower than 0.1 per cent (i.e. with a $z>z_{\mathrm{FAP}}=0.1$ per cent) are statistically significant, while for those with an FAP between 
Table 5. Offsets betweenspectral and photometric indices data sets from different instruments.

\begin{tabular}{|c|c|c|c|c|c|}
\hline Instruments & $\mathrm{H} \alpha$ Offset & CaHK Offset & NaD Offset & $\begin{array}{l}\text { FWHM Offset } \\
\quad\left(\mathrm{km} \mathrm{s}^{-1}\right)\end{array}$ & $m_{\mathrm{V}}$ Offset (mag) \\
\hline HIRES-HARPSpre & $0.00198 \pm 0.00006$ & $0.4817 \pm 0.0006$ & - & - & - \\
\hline HIRES-APF & $-0.024 \pm 0.002$ & $-2.276 \pm 0.007$ & - & - & - \\
\hline HIRES-UVES & $0.05624 \pm 0.00006$ & - & - & - & - \\
\hline CARMENES-HARPSpost & $-0.01547 \pm 0.00002$ & - & $-0.00678 \pm 0.00007$ & $0.12888 \pm 0.00001$ & - \\
\hline CARMENES-HARPSN & $-0.03559 \pm 0.00003$ & - & $-0.00412 \pm 0.00005$ & $0.20562 \pm 0.00001$ & - \\
\hline HARPSpre-PFS & - & - & $0.0250 \pm 0.0002$ & - & - \\
\hline HARPSpre-APF & - & - & $-0.075 \pm 0.002$ & - & - \\
\hline ASAS-FCAPT+RCT & - & - & - & - & $-0.00870 \pm 0.00002$ \\
\hline ASAS-AAVSO & - & - & - & - & $-0.01531 \pm 0.00002$ \\
\hline ASAS-SNO & - & - & - & - & $-0.011340 \pm 0.000007$ \\
\hline
\end{tabular}

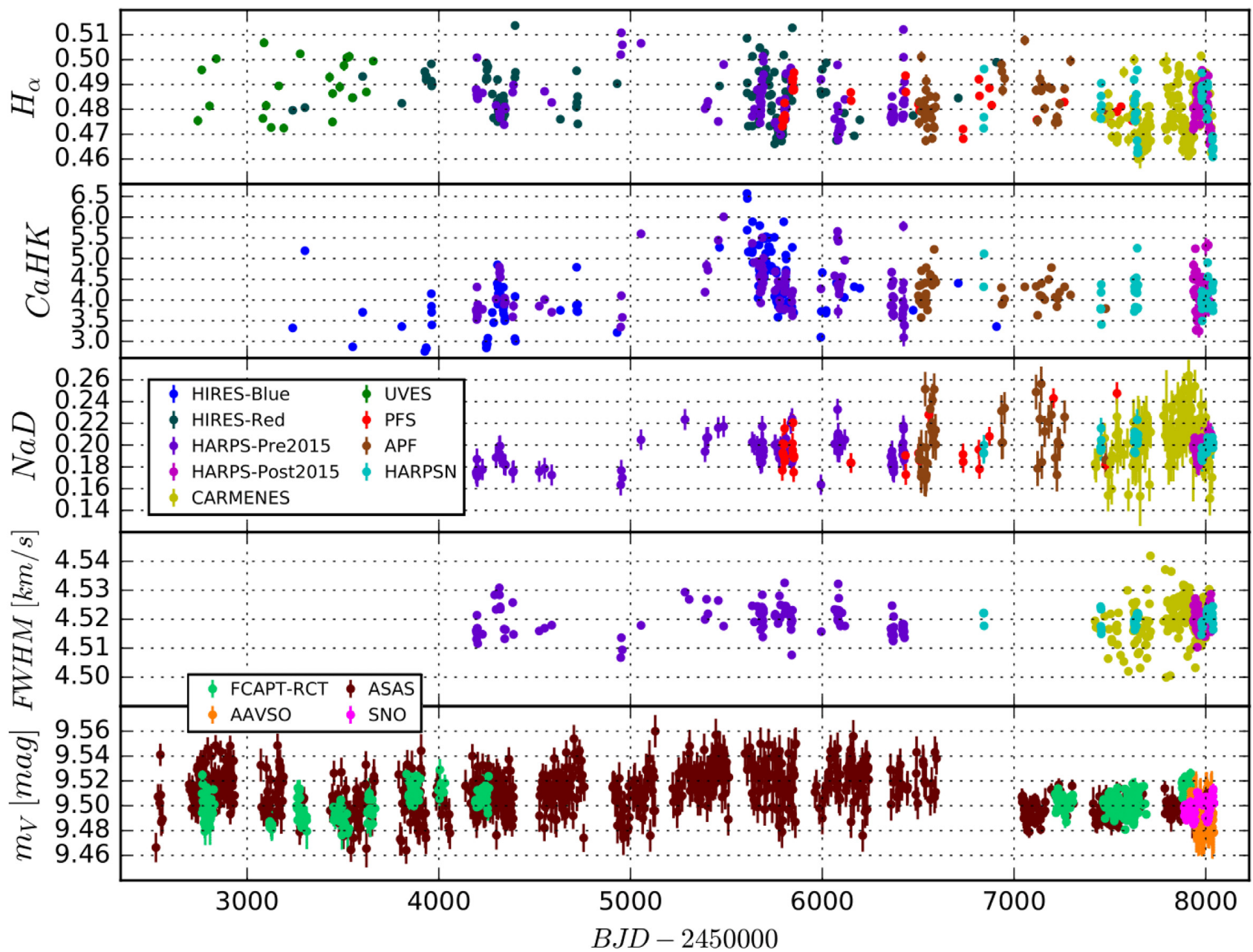

Figure 5. Time series of the four spectroscopic indexes and the $V$ photometry with their respective offsets applied. The NaD plot contains a legend with all the spectrographs and the FWHM plot contains a legend with the instruments used for the photometry analysis.

0.1 percent and 10 percent we cannot ensure that they are not false positives, and therefore we may discuss some signals, in particular, those below or close to an FAP of 1 per cent as tentative signals.

We first carried out a pre-whitening process for a single instrument. We started calculating the periodogram with the FAP levels from bootstrapping. We selected a signal from the periodogram (usually the most significant one) and modelled it with a double sinusoidal fit to subtract that signal and, thus, recompute the periodogram (Boisse et al. 2011). The double sinusoidal fit is defined as

$y(t)=A_{1} \times \sin \left(\omega_{1}+\phi_{1}\right)+A_{2} \times \sin \left(\omega_{2}+\phi_{2}\right)+A_{3}$,

where $\omega_{2}=2 \omega_{1}=2 \pi f / P$. We left $A_{1}, A_{2}, A_{3}, \phi_{1}, \phi_{2}$, and $P$ as free parameters, restricting the value of $P$ in a 15 percent 


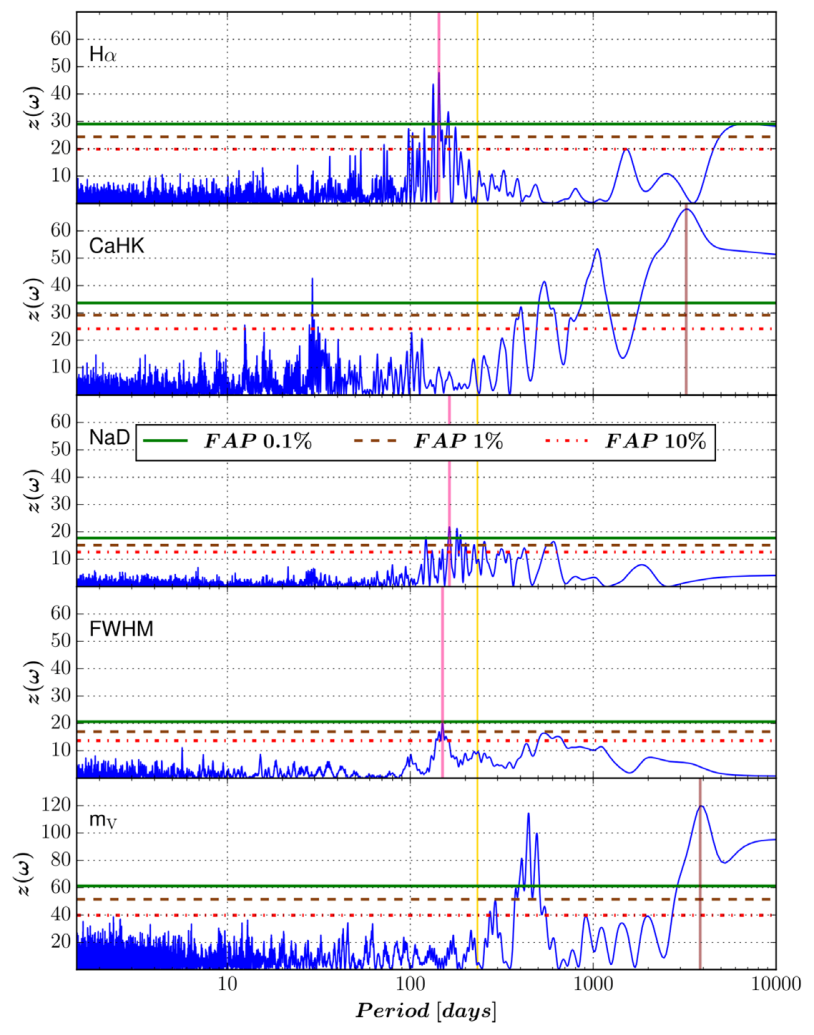

Figure 6. Periodograms of the four spectroscopic indexes and the $V$-band photometry using the values from Fig. 5. The most significant signals have been marked in different colours: pink for the ones associated with the rotation period and brown for the ones associated with the long-term activity cycle. The vertical yellow line shows the period of the recently discovered super-Earth Barnard b (Ribas et al. 2018).

from the original period in the periodogram. We used this double sinusoidal model in order to account the asymmetry of some signals (Berdyugina \& Järvinen 2005) with the MPFIT routine (Markwardt 2009). We also add a jitter term associated with every individual instrument present in the complete data set to this model in order to account possible bad estimations in the index errors, along with a trend correction if we detect long-term variations above our time coverage. After subtracting the first signal, we repeated the process (maintaining the same FAP levels) until we had no more significant signals in the periodogram.

After the pre-whitening process we isolated each individual signal from the rest of signals that we subtracted along this process. We selected one signal at a time and used the frequencies from the rest to make a model. The subtraction of this model gave us an isolated periodogram, where we can check that the original period was not caused by effects of the other signals. We obtain this isolated periodogram for every single signal that was detected along the prewhitening process.

Once we carried out the whole process for one single instrument, we added a second instrument with its respective offset and repeated the modelling-subtraction-isolation method, because the information provided by a single instrument may not be enough in terms of time-span or sampling. The addition of instruments follows the order shown in Table 6: we analysed each block of instruments separately and then join them. When we combined these two blocks of instruments, we needed to estimate an additional offset using a wider time window $(30 \mathrm{~d})$. These offsets are also shown in Table 6 .
Finally, we computed the window function for each time series of each activity indicator including the photometric and RV data using the systemic console (Meschiari et al. 2009). We find only a few signals related to the daily sampling and the yearly periodicity of the observations (the most significant at 365 and $1850 \mathrm{~d}$ ).

\section{ANALYSIS}

In this section, we describe the analysis and results of each activity indicator. In the spectroscopic analysis, we have used all data sets available according to Table 4 . In the photometric analysis we use again all data sets given in Table 4 (see more details in Section 4.5). All the results shown in this section has been done following the methodology described in Section 3.

\section{1 $\mathrm{H} \alpha$ index}

In the case of $\mathrm{H} \alpha$ we obtained 619 measurements, characterized by an average of 0.48 , mean error of 0.001 , and rms of 0.01 . We began analysing the HIRES data set (in the HIRES-Red configuration), and then added individually HARPS-Pre2015, PFS, APF, and UVES. After every addition, we used the modelling-subtraction technique to see which signals are hidden behind the main ones. We repeated this treatment for the second block of instruments, beginning with HARPS-Pre2015 and adding HARPS-N and CARMENES in that particular order. When we combined the two blocks, the periodogram of the data gives a $7692 \mathrm{~d}$ signal as the second most significant peak after the $\sim 140-150 \mathrm{~d}$ peak (see the first periodogram of Fig. 6). To see if this signal was caused by any instrumental effect, we applied a trend correction to the whole data set, and this signal disappeared, as it is shown in the top of Fig. 7.

After the trend correction, the most significant peak is at $143 \mathrm{~d}$, which is close to the rotation period determined by Suárez Mascareño et al. (2015). This signal is surrounded by multiple peaks between 130 and $177 \mathrm{~d}$ with low FAP. We fit this forest of peaks with a Gaussian model, whose FWHM gives us an error associated with the $143 \mathrm{~d}$ signal of $15 \mathrm{~d}$. We note that the baseline of the observations is much longer than the expected lifetime of spots and plages on the surface of the star. These magnetic phenomena can occur at different stellar latitudes, favouring these multiple peaks around the rotation signal (see Section 5). In the second periodogram of Fig. 7, the second signal detected, after the subtraction of the $143 \mathrm{~d}$ signal (modelled by a double sinusoidal), has a $149 \mathrm{~d}$ period with an FAP close to the 1 percent level. When we isolate the first signal from the second one, the highest peak stays at $143 \mathrm{~d}$ with an amplitude of $0.00523 \pm 0.00001$ and an FAP above the 0.1 per cent, as shown in Fig. 8.

To complement this analysis, we introduce one jitter term for every single spectrograph in the double sinusoidal model and we change the independent term $\left(A_{3}\right)$ for a linear trend term $\left(A_{3}+A_{4} \mathrm{x}\right.$ $t$ ), which leads to a very similar pre-whitening process shown in the Fig. 9. In this case, the error re-calculation associated with the jitter terms produce that the second signal to be detected shifts to $177 \mathrm{~d}$ with an FAP close to the 1 per cent level, and may also be related with differential rotation. The forest of peaks around the rotation period in the residuals is similar to the one shown in the bottom panel of Fig. 7, giving us a rotation range between 130 and $180 \mathrm{~d}$.

\subsection{Ca II HK index}

In the time series of the CaHK index, the blue arm spectra of UVES, where the calcium lines are located, was not available. 
Table 6. Additionorder of spectrographs for each individual index and offsets between spectral indices data sets from different blocks of spectrographs.

\begin{tabular}{lccc}
\hline Index & Block & Spectrograph & Offset \\
\hline $\mathrm{H} \alpha$ & 1 & HIRES-HARPSpre-PFS-APF-UVES & $0.0145 \pm 0.0002$ \\
& 2 & CARMENES-HARPSpost-HARPSN & \\
$\mathrm{CaHK}$ & 1 & HIRES-HARPSpre-APF & $0.62 \pm 0.02$ \\
& 2 & HARPSpost-HARPSN & \\
$\mathrm{NaD}$ & 1 & HARPSpre-PFS-APF & $0.0226 \pm 0.0006$ \\
& 2 & CARMENES-HARPSpost-HARPSN & \\
FWHM & 1 & HARPSpre & $-0.003114 \pm 0.000001$ \\
& 2 & CARMENES-HARPSpost-HARPSN & \\
\hline
\end{tabular}

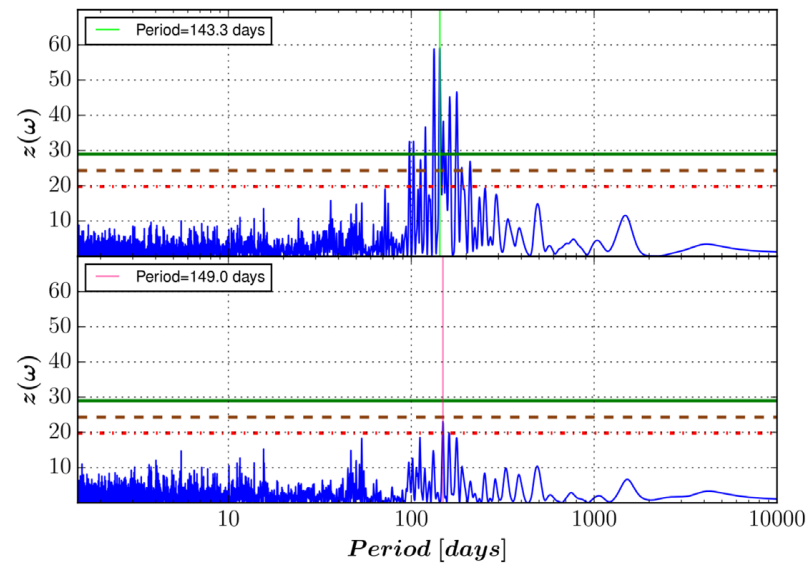

Figure 7. Top: Periodogramsof the time series of $\mathrm{H} \alpha$ for HARPS+HARPSN+CARMENES+HIRES+APF+PFS+UVES spectra after the trend correction. Bottom: Periodogram of the residuals after the subtraction of the $143 \mathrm{~d}$ period signal.
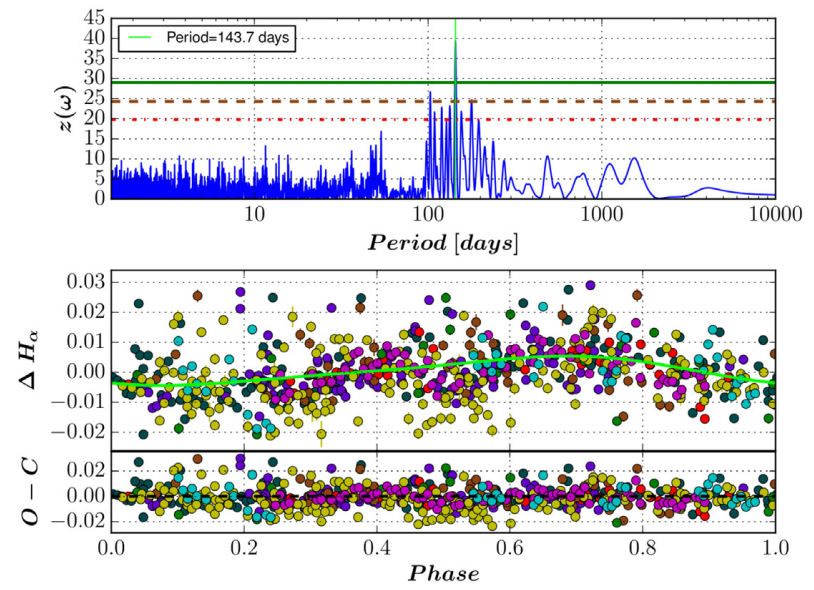

Figure 8. Top: Periodogram of the time series of $\mathrm{H} \alpha$ index after the subtraction of the 149 d period signal. Bottom: Phase-folded curve of the $\mathrm{H} \alpha$ time series using the $143 \mathrm{~d}$ period. Each spectrograph has been represented with a different colour, following the legend in Fig. 5. The green line represents the best double-sinusoidal fit found by the MPFIT routine.

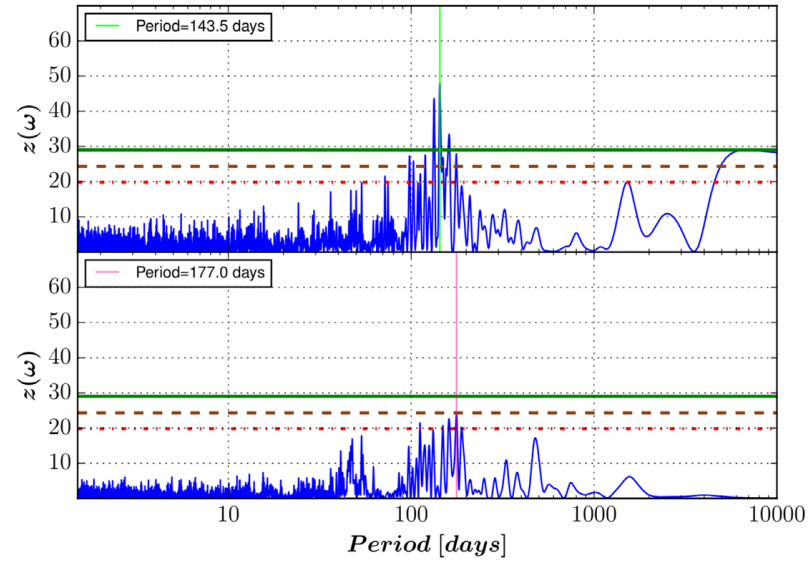

Figure 9. Top: Periodograms of the time series of $\mathrm{H} \alpha$ for HARPS+HARPSN+CARMENES+HIRES+APF+PFS+UVES spectra. Bottom: Periodogram of the residuals after the subtraction of the $143 \mathrm{~d}$ period signal with a double sinusoidal model including jitter terms and a linear trend.

The CARMENES wavelength coverage did neither include the Ca II H\&K spectral range, and therefore it was not used. We also omitted PFS due to the high noise in that wavelength range. Therefore, we had 385 measurements of this index coming from HARPS, HARPS-N, HIRES, and APF, with an average of 4.63, mean error of 0.06 , and rms of 0.6 . Owing to the new continuum filters introduced in this work, we did not use the Mount Wilson calibration (Vaughan, Preston \& Wilson 1978) for this index. Using the four time series with their respective offsets and without the Mount Wilson calibration, we first detected a $3225.8 \mathrm{~d}$ signal that remains stable after the trend subtraction, as it is shown in Fig. 10. This long-period signal may be related to a long-term activity cycle in the star. It has an FAP level above the 0.1 per cent and its fitted by the double sinusoidal shown in Fig. 11 that includes jitter terms and has an amplitude of $0.5 \pm 0.4$. When we subtract this model, we obtain a $120 \mathrm{~d}$ period signal with very low significance that seems to be dependent on the model used to subtract the long-term signal. Depending on the use of jitter terms and trend correction, we obtain different peaks in the range from $\sim 80$ to $200 \mathrm{~d}$ with a similar FAP, so we could not ensure that any of the signals are indeed stellar activity signals. We also could not find a clear signal associated with the expected rotation in the analysis of the CaHK index. 


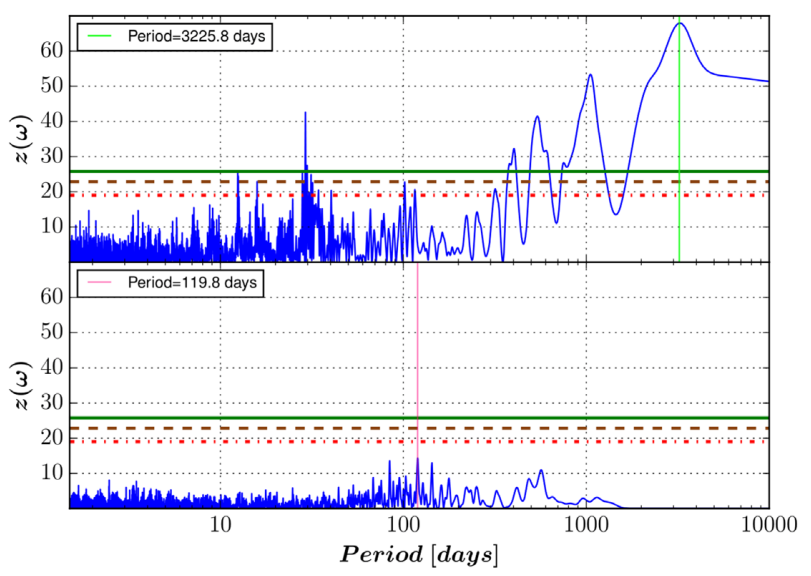

Figure 10. Top: Periodograms of the time series of CaHK for HIRES-Blue+HARPS-Pre2015+APF+HARPS-Post2015+HARPSN spectra. Bottom: Periodogram of the residuals after the subtraction of the $3225.8 \mathrm{~d}$ period signal.

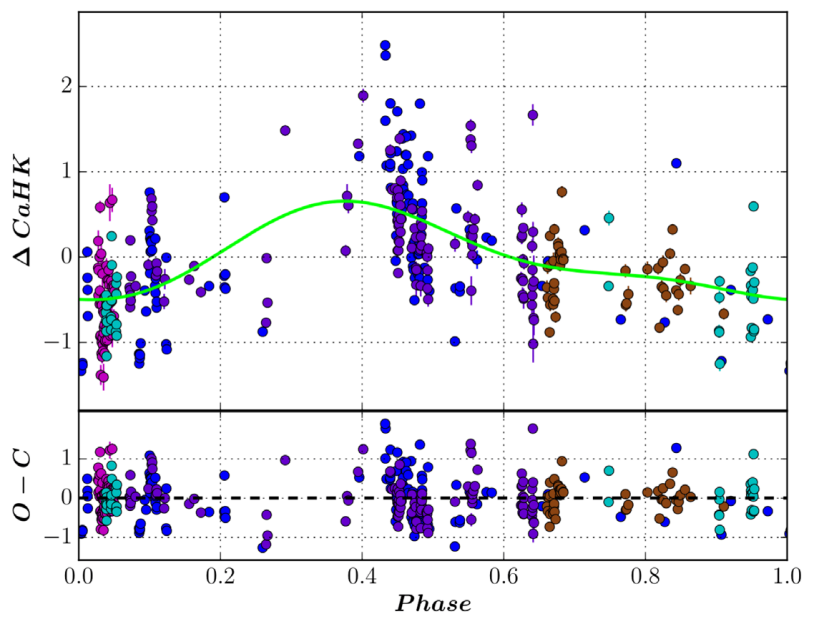

Figure 11. Phase-folded curve of the CaHK time series using the $3226 \mathrm{~d}$ period. Each spectrograph has been represented with a different colour, following the legend in Fig. 5. The green line represents the best doublesinusoidal fit found by the MPFIT routine.

\subsection{Na I D index}

The time-series measurements of the Na I D index do not include HIRES data because we could not get a reliable wavelength calibration for the Echelle orders that contains the core lines and the continuum regions. We also avoid using UVES due to the lower $\mathrm{SN}$ ratio in those orders. This leaves 453 measurements with an average of 0.19 , mean error of 0.01 , and rms of 0.02 . When we treat this time series and combine the two blocks of instruments we obtain the first periodogram shown in Fig. 12. We found a signal at $164 \mathrm{~d}$ surrounded by a forest of peaks similar to the one found in $\mathrm{H} \alpha$ that could be associated with the rotation of the star. The difference in period with respect to the detected signal in the $\mathrm{H} \alpha$ index suggests that this signal could be caused by differential rotation. In the Sun, for instance, the rotation period can vary from the equator ( $25 \mathrm{~d}$ ) to the pole ( $35 \mathrm{~d}$ ) in 40 per cent. From a sample of more than 24000 active Kepler stars, Reinhold et al. (2013) found evidences of differential rotation within the 30 per cent of the equatorial rotation period in 77 percent of the sample. In a more recent study, Aigrain et al. (2015) tested a blind hare-and-hounds

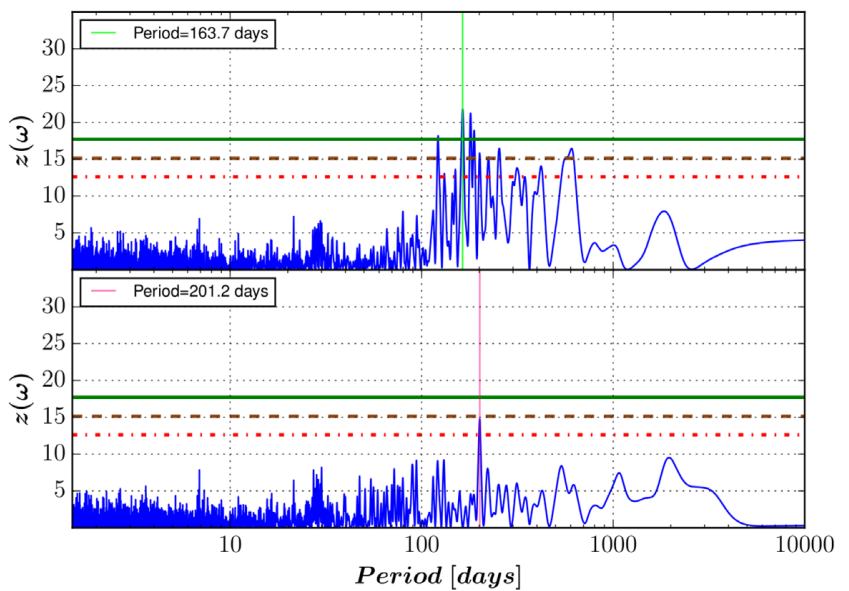

Figure 12. Periodograms with the detected signals in the time series of $\mathrm{NaD}$ for HARPS-Pre2015+PFS+APF+CARMENES+HARPSPost2015+HARPSN spectra using the pre-whitening technique.

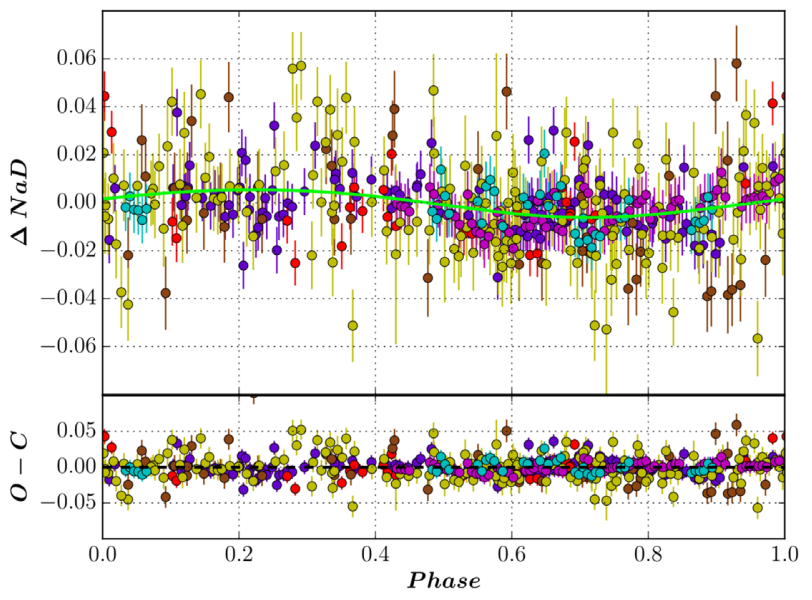

Figure 13. Phase-folded curve of the $\mathrm{NaD}$ time series using the $164 \mathrm{~d}$ period. Each spectrograph has been represented with a different colour, following the legend in Fig. 5. The green line represents the best doublesinusoidal fit found by the MPFIT routine.

exercise using 1000 simulated photometric light curves, and found little correlation between the reported and simulated values of the differential rotation, indicating that this detection in single light curves must be treated with caution. With a spectroscopic study like ours, using time series from different activity indicators, we gain reliability with a detection of the same structure around the rotation period in two of the time series. In this case, the variation from the original period measured in $\mathrm{H} \alpha$ to the one measured in $\mathrm{Na}$ I D is only 15 percent. This signal has an amplitude of $0.0070 \pm 0.0008$ and its FAP grows near the 0.1 percent. When we subtract this signal with the double sinusoidal model shown in Fig. 13 that includes jitter terms, the rest of the peaks remain with higher FAP values than 1 percent, and they may be caused by the offsets between spectrographs, so no more clear information was extracted from this index.

\subsection{Full width half maximum}

Finally, the time series of the FWHM, consists of 393 measurements, with an average of $4.52 \mathrm{~km} \mathrm{~s}^{-1}$, mean error of 


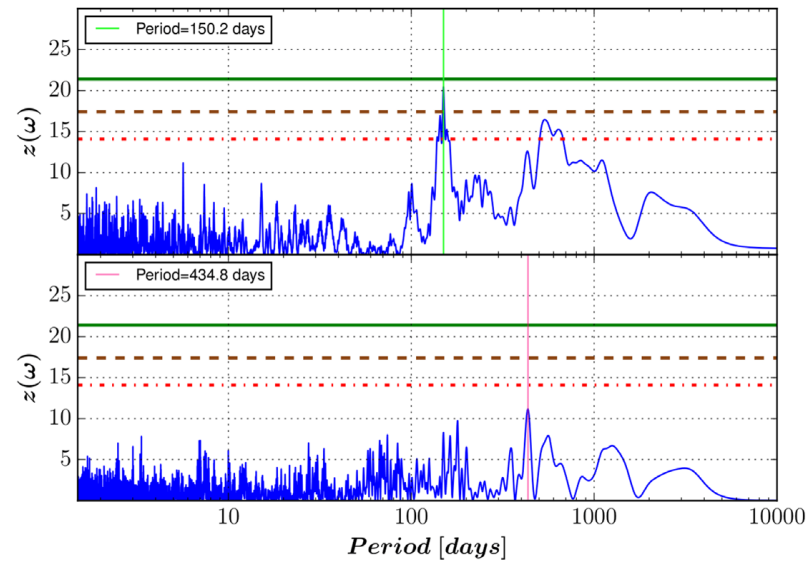

Figure 14. Top: Periodograms of the time series of FWHM for HARPSPre2015+CARMENES+HARPS-Post2015+HARPSN spectra. Bottom: Periodogram of the residuals after the subtraction of the $150 \mathrm{~d}$ period signal.

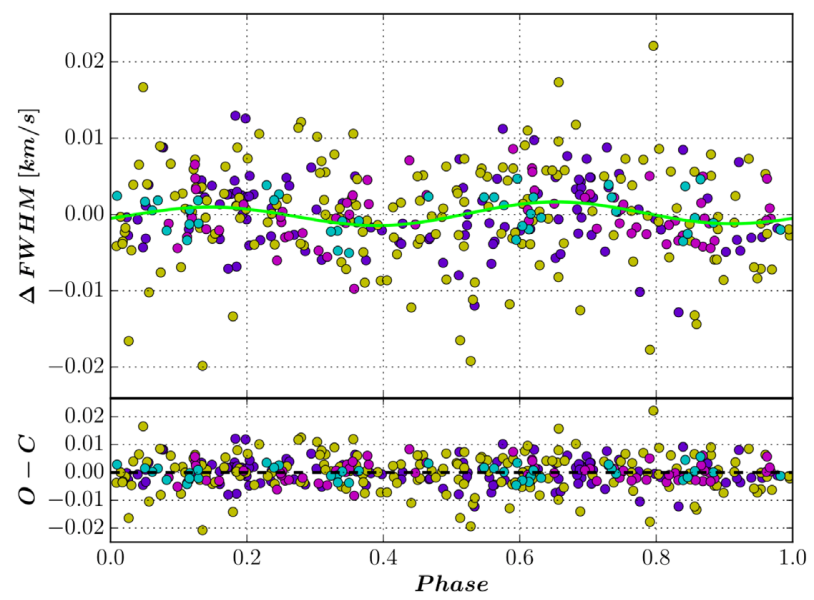

Figure 15. Phase-folded curve of the FWHM time series using the $150 \mathrm{~d}$ period. Each spectrograph has been represented with a different colour, following the legend in Fig. 5. The green line represents the best doublesinusoidal fit found by the MPFIT routine.

$0.00005 \mathrm{~km} \mathrm{~s}^{-1}$, and $\mathrm{rms}$ of $0.006 \mathrm{~km} \mathrm{~s}^{-1}$. We first apply a trend correction to the HARPS-Pre2015 values due to a focus drift problem. We also noticed a highest dispersion in the CARMENES values (see Fig. 5) that may be related with the lack of weights per order in this spectrograph (the CCFs that we have used were already built as a one average function). The combination of the three spectrographs for which we have a CCF leads to a tentative detection of the rotation period at $150 \mathrm{~d}$ with an FAP level close to the 0.1 per cent, as it is shown in Fig. 14. In this case, the signal is fitted by the double sinusoidal shown in Fig. 15with an amplitude of $0.00343 \pm 0.00006 \mathrm{~km} \mathrm{~s}^{-1}$. After subtracting this first peak with a double sinusoidal including jitter terms and a global trend, the remaining peaks do not exceed the 10 per cent level of FAP, making it difficult to establish a clear origin for them.

\subsection{Photometry}

We complement our spectroscopic analysis using the time series of $V$-band photometric measurements. A higher number of data points are available (1390) compared to the spectroscopic data set (619 as maximum), with an average of $9.5 \mathrm{mag}$, mean error of $9.2 \mathrm{mmag}$,

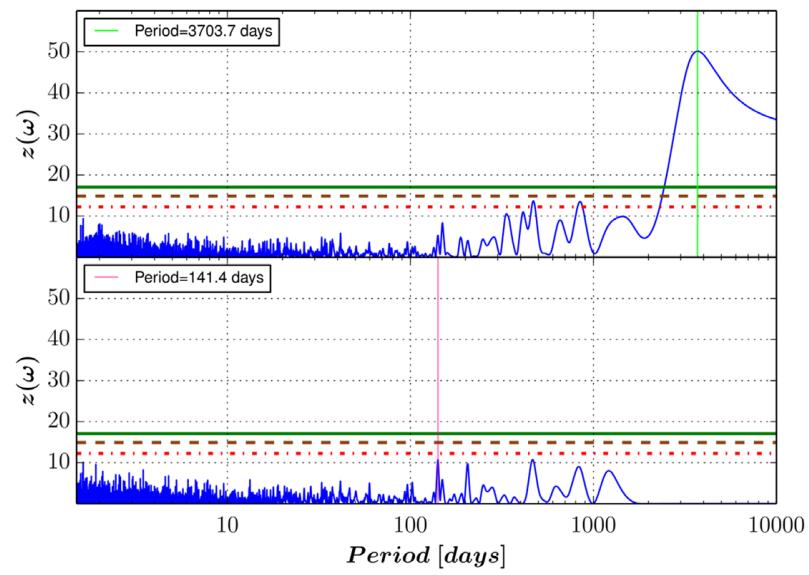

Figure 16. Top: Periodogram of the time series of ASAS-S+ASAS-N $m_{V}$. Bottom: Periodogram of the residuals after the subtraction of the $3703.7 \mathrm{~d}$ period signal.

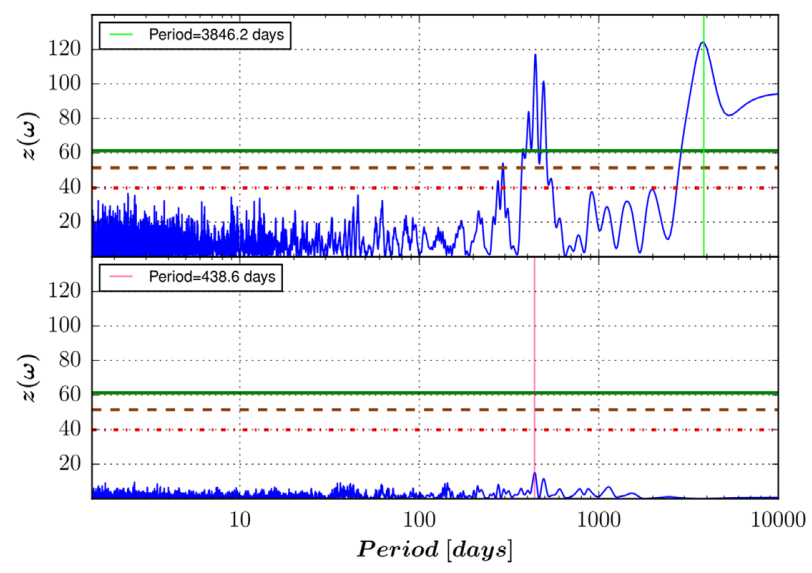

Figure 17. Top: Periodogram of the time series of ASAS+AAVSO+SNO $m_{V}$. Bottom: Periodogram of the residuals after the subtraction of the $3846 \mathrm{~d}$ period signal.

and rms of 15.4 mmag. We begin by analysing the largest data set (ASAS), combining the ASAS-S and ASAS-N time series.

As it is shown the top panel in Fig. 16, we find the first signal at $3703.7 \mathrm{~d}$, which may be related to a long-term activity cycle in the star. After the subtraction of this signal with a very high amplitude $(0.012 \pm 0.004 \mathrm{mag})$, the rest of the peaks in the periodogram remain under the 10 percent level of FAP, with the rotation period at $141 \mathrm{~d}$ being the second signal in amplitude. The addition of the ASAS-SN data set produces a shift in the peak of the long-period signal to $3846 \mathrm{~d}$, increasing its amplitude, and also producing an increase in the FAP levels from the bootstrapping.

When we add the AAVSO and SNO data sets and determine the offsets using ASAS as reference, we recover the long-term activity cycle signal at $3846 \mathrm{~d}$ signal present in the complete ASAS data set as it shows the first periodogram of Fig. 17, where the bootstrapping process to obtain the FAP levels was done omitting the ASAS-SN data. The double sinusoidal model that includes jitter terms and a linear trend presents an amplitude of $0.012 \pm 0.006$ mag, and its subtraction produces the periodogram shown in the bottom panel of Fig. 17, where the peak with the highest amplitude is a signal at $438.6 \mathrm{~d}$ with very low significance. 

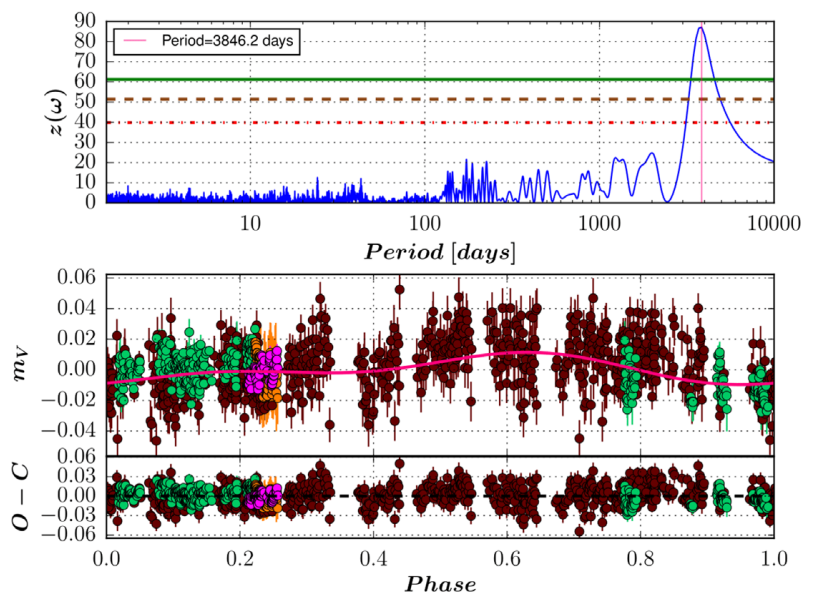

Figure 18. Top: Periodogram of thetime series of ASAS + AAVSO + SNO+FCAPT - RCT $m_{\mathrm{V}}$ after the subtraction of the 204.5 d period signal. Bottom: Phase-folded curve of the $m_{V}$ time series using the $3846.2 \mathrm{~d}$ period. Each instrument has been represented with a different colour, following the legend in Fig. 5. The pink line represents the best double-sinusoidal fit found by the MPFIT routine.

The addition of the FCAPT-RCT data set (see Fig. 18) creates a broad and signal at $204.5 \mathrm{~d}$ as the most significant one. After the subtraction of this first signal, we recover the long-term activity cycle signal at $3846.2 \mathrm{~d}$ obtained in previous time series with an amplitude of $0.009 \pm 0.008$ mag. This signal is shown in Fig. 18, where the periodicity of the cycle is even more clear than in the time series of the $\mathrm{CaHK}$ index due to the higher number of points. The FAP values come from the bootstrapping process carried out omitting the ASAS-SN and FCAPT-RCT values.

The problem with the FCAPT-RCT data set is the underestimation in the $m_{\mathrm{V}}$ errors, which produces the high-amplitude signal at $204.5 \mathrm{~d}$ in the periodogram along with higher FAP values from the bootstrapping process that can make the long-term activity cycle signal be confused with noise because it does not reach the 10 per cent level of FAP. When we apply a jitter term to this data set and recalcute the errors, we recover the long-term signal with its expected amplitude. It is also important to take into account the time gap of $\sim 8$ yr between the FCAPT and RCT data sets, which can affect the results due to a bigger uncertainity in the offset between those two data sets. The same happens between the ASASS+ASAS-N and ASAS-SN data set, with a gap of more than $1 \mathrm{yr}$ between them, but in this case the difference is not so remarkable. We maintain the ASAS-SN time series in the analysis because it is needed to obtain the offset values using the time windows methodology and the FCAPT-RCT time series because it increases the $m_{V}$ amplitude of the long-term activity cycle signal.

In the separate analysis of Montsec and MEarth time series from different photometric filters, we do not detect any significant signal that could be attributed to rotation or a long-term activity cycle, although MEarth has proven to be capable of detecting rotation periods Newton et al. (2016a).

\subsection{Chromatic index and bisector span}

We also did an additional analysis using the time series of the CRX that contains 216 measurements taken by CARMENES in a $2 \mathrm{yr}$ time span. This activity indicator was defined by Zechmeister et al. (2018) and it serves to measure the RV-wavelength dependence.
The CARMENES pipeline correlates these two quantities along all the Echelle orders and then fits a first-order polynomial whose slope is taken as a measurement of the CRX.

In the time series of this index, we first found a $10000 \mathrm{~d}$ signal that dissapears after a trend correction. This hints to the presence of a change in the level of activity on time-scales much larger than the range of our observations. After the correction, the FAP of the most significant signal is greater than 10 percent, so we could not find anything relevant in this time series.

We also analysed the bisector span time series (BIS), an index that comes from the slope of the polynomial that fits the centroid of the CCF at different heights (Queloz et al. 2001). The BIS time series is composed by 116 measurements of HARPS-N, 31 of HARPS-N, and 186 of CARMENES. This index measures the distortion of the CCF under the presence of stellar spots and plages. This distortion is lower in fast rotators and low activity stars. As for the CRX index, we could not find any significant signal in the analysis of the BIS time series.

\section{DISCUSSION}

Combining the rotation period from $\mathrm{H} \alpha$ and FWHM time series with weights according to their FAP level we obtain a final average value of $145 \pm 15 \mathrm{~d}$. This 10 per cent error comes from the FWHM of a Gaussian model that fits the forest of peaks around the $145 \mathrm{~d}$ signal and takes into account the uncertainty in the latitude of the active regions that are producing this signal. This means that Barnard's Star is among the main-sequence stars with lowest rotation known to date, above the M-stars average periods (Newton et al. 2016a; Suárez Mascareño et al. 2018b). This also suggests that Barnard's age matches the age of the local thick disc (Newton et al. 2018).

Differential rotation may be responsible for the different signals found in between 130 and $180 \mathrm{~d}$, as a consequence of the presence of active regions at different latitudes of the stellar surface. This phenomenon has not been fully understood for stars from all spectral types, but especially for M-dwarfs. Reinhold \& Gizon (2015) confirmed a relation between rotation period and differential rotation predicted by Reiners \& Schmitt (2003) including M-type stars in their sample. Although this relation has only been proven for stars with $\mathrm{P}_{\min }<50 \mathrm{~d}$, we obtain a value for Barnard's Star of $\alpha=\left(P_{\max }-P_{\min }\right) / P_{\max }=0.278$ that matches the M-stars values present in this study. Taking into account that differential rotation is more evident in slow rotators, we conclude that our estimation is consistent with the theoretical prediction for differential rotation.

We detected two similar long-period signals, in the Ca II H\&K and $m_{V}$ time series. The two signals show similar periodicities, both compatible with the length of a solar-like cycle. When we compare the two series side by side (see Fig. 19) we can see even more similarities. Not only their periods are compatible, but also the dates of their maxima and minima are virtually the same, hinting a common underlying phenomenon. The combination of the two series gives us coverage along two full phases of the signal, pointing at a cyclic nature. We can interpret this variability as the footprint of a magnetic cycle of $10 \pm 2 \mathrm{yr}$, which is not expected for a completely convective star like Barnard's Star (Chabrier \& Kürster 2006; Wargelin et al. 2017).

It is interesting to note that the position of the maximum emission phase coincides with the position of the faintest phase of the star and the minimum emission phase coincides with the brightest phase of the light curve. Given the low level of chromospheric and X-ray emission of Barnard's Star (Passegger et al. 2018), this behaviour is opposite to the solar case, and to most old FGK stars (Radick et al. 


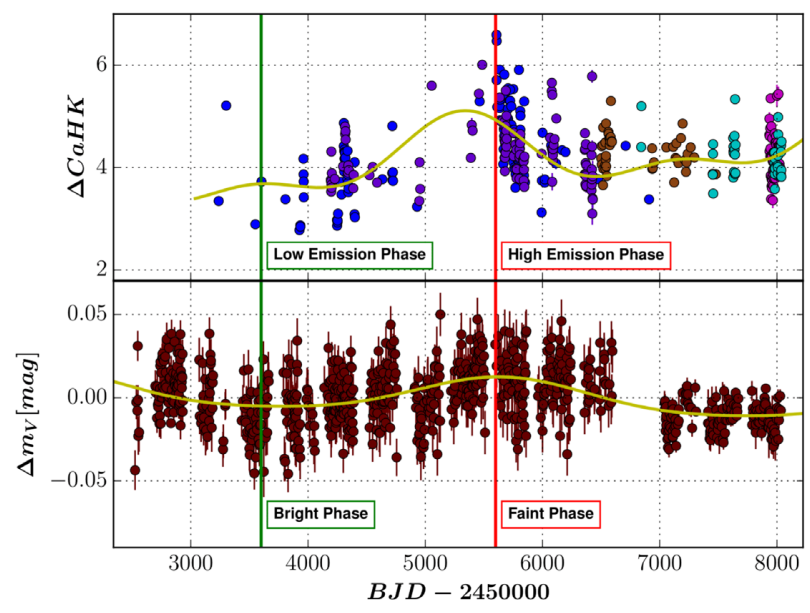

Figure 19. Top: Time seriesof CaHK for HIRES-Blue+HARPSPre2015+APF+HARPS-Post2015+HARPSN spectra. The beginning of the low-emission phase is marked in green and the beginning of the high emission is marked in red. Bottom: Time series of ASAS-S+ASAS-N $m_{\mathrm{V}}$. The beginning of the bright phase is marked in green and the beginning of the faint is marked in red. In both panels, the yellow line represents the best double-sinusoidal fit found by the MPFIT routine.

Table 7. Semi-amplitudeof the isolated signals from the four spectroscopic indexes and the photometric magnitude.

\begin{tabular}{lcc}
\hline Index & $P(\mathrm{~d})$ & Semi-amplitude \\
\hline $\mathrm{H} \alpha$ & 143.68 & $0.00523 \pm 0.00001$ \\
& 149.03 & $0.00305 \pm 0.0001$ \\
$\mathrm{CaHK}$ & 3225.81 & $0.5 \pm 0.4$ \\
$\mathrm{NaD}$ & 163.67 & $0.0070 \pm 0.0008$ \\
$\mathrm{FWHM}$ & 150.15 & $0.00343 \pm 0.00006 \mathrm{~km} \mathrm{~s}^{-1}$ \\
$m_{V}$ & 3846.15 & $0.009 \pm 0.008 \mathrm{mag}$ \\
\hline
\end{tabular}

1998). It would be compatible with a spot-dominated stellar surface, typical of active FGK stars. In active stars, spots dominate the brightness changes, while plages would dominate chromospheric and X-ray emission. The situation is similar to what (Wargelin et al. 2017) found for the case of Proxima, when comparing $V$-band photometry to X-ray and UV emission. Despite being old, Proxima remains quite active (Pavlenko et al. 2017), which made it natural to put in on the 'active stars' category. The case of Barnard is quite different, as the star shows very low levels of chromospheric and X-ray emission. This could hint at late M-dwarfs keeping the 'active star' behaviour, and remaining spot dominated, even after their chromospheric and X-ray emission reach extremely low levels.

Given our short baseline, the exact period and long-term behaviour are still complicated to asses. Further monitoring spectroscopic and photometric would be needed to better characterize it.

The amplitude of the rotation period and long-term activity cycle signals is shown in Table 7.

Applying the Mount Wilson calibration to the $S$-index of all spectrographs by the following expression:

$S_{m w}=\alpha \cdot S+\beta$,

where $\alpha=1.111, \beta=0.0153$ (Lovis et al. 2011), and $S$ is calculated with the original passbands, we can use its mean value $<S_{\mathrm{mw}}>$ to calculate the level of chromospheric activity $\log _{10}\left(\mathrm{R}_{\mathrm{HK}}^{\prime}\right)$ as (Noyes et al. 1984):

$\log _{10}\left(R_{\mathrm{HK}}^{\prime}\right)=\log _{10}\left(\left(1.34 \times 10^{-4}\right) \cdot C_{c f} \cdot<S_{\mathrm{mw}}>-R_{\mathrm{phot}}\right)$,

where $C_{\mathrm{cf}}$ is a conversion factor to correct the flux variations in the continuum passbands and also to normalize to the bolometric luminosity, that is defined as (Suárez Mascareño et al. 2015):

$$
\begin{aligned}
\log _{10}\left(C_{\mathrm{cf}}\right)= & -0.443-0.645(B-V)-1.270(B-V)^{2} \\
& +0.668(B-V)^{3}
\end{aligned}
$$

and $R_{\text {phot }}$ is the photospheric contribution to the calcium core lines (Hartmann et al. 1984) that we need to get rid of in order to measure only the chromospheric contribution

$\log _{10}\left(R_{\text {phot }}\right)=\left(1.48 \times 10^{-4}\right) e^{-4.3658(B-V)}$.

Equation (9) gives a chromospheric activity level of $\log _{10}\left(R_{\mathrm{HK}}^{\prime}\right)$ $=-5.82 \pm 0.08$ using our $S_{\mathrm{mw}}$ measurements of Gl 699 that is in good agreement with the values of -5.69 (Astudillo-Defru et al. 2017) and -5.86 (Suárez Mascareño et al. 2015) from the literature. If we use the relation between the chromospheric activity level and induced RV semi-amplitude found by Suárez Mascareño et al. (2017, 2018b), we get an induced semi-amplitude of $K=0.67_{-0.20}^{+0.28} \mathrm{~m} \mathrm{~s}^{-1}$, which give us an upper limit of $0.95 \mathrm{~m} \mathrm{~s}^{-1}$ that marginally falls on the detection limit for most of the current instrumentation dedicated to RV searches (Pepe, Ehrenreich \& Meyer 2014).

In the last years, several groups have studied the rotation periods of a large sample of stars (see e.g. McQuillan, Mazeh \& Aigrain 2014; Newton et al. 2016a; Díez-Alonso et al. 2019). We have selected a sample from Suárez Mascareño et al. (2015, 2016, 2018a,b) and Astudillo-Defru et al. (2017), to see how the values of the rotation period and the level of chromospheric activity obtained for Barnard's Star fit into the relation found by Suárez Mascareño et al. (2016)

$\log _{10}\left(P_{\mathrm{rot}}\right)=A+B \cdot \log _{10}\left(R_{\mathrm{HK}}^{\prime}\right)$,

where $A=-2.37 \pm 0.28$ and $B=-0.777 \pm 0.054$ for M-type stars with a $\log _{10}\left(R_{\mathrm{HK}}^{\prime}\right) \leq-4.1$ (Suárez Mascareño et al. 2018b). As it is shown in Fig. 20, our rotation period fits very well into this theorical prediction (the rotation period value given by this relation is $142 \mathrm{~d}$ ).

Also previous studies (Pizzolato et al. 2003; Wright et al. 2011; Reiners, Schüssler \& Passegger 2014; Wright \& Drake 2016) have investigated the relation between the $\mathrm{X}$-ray emission and the rotation rate of stars. Using the relation found by Wright et al. (2011) we estimate a rotation period of $132 \mathrm{~d}$, which is consistent with our result.

In the photometric time series, we found the rotation period at $141 \mathrm{~d}$ period in the ASAS subset of data (that spans about $15 \mathrm{yr}$ ), although with low significance [FAP $<10$ per cent, similar to the level obtained by Benedict et al. (1998) with the HST data]. A period of $\sim 200 \mathrm{~d}$ is also present when we add the FCAPT-RCT data set. This data set has a time coverage similar to ASAS (14 yr), but with much sparse (about a third of the ASAS data, 348-836 epochs) and uneven gapped data, so that the offset could be responsible for the discrepancies in the photometric results, or less likely, differential rotation, as $180 \mathrm{~d}$ would be $\sim 30$ percent of the estimated $140 \mathrm{~d}$ rotation period.

The year-alias of a rotation period of $145 \mathrm{~d}$ of happens at a period of about $240 \mathrm{~d}$ (Dawson \& Fabrycky 2010) that is close to the planetary signal of $233 \mathrm{~d}$ of Barnard b (Ribas et al. 2018). 


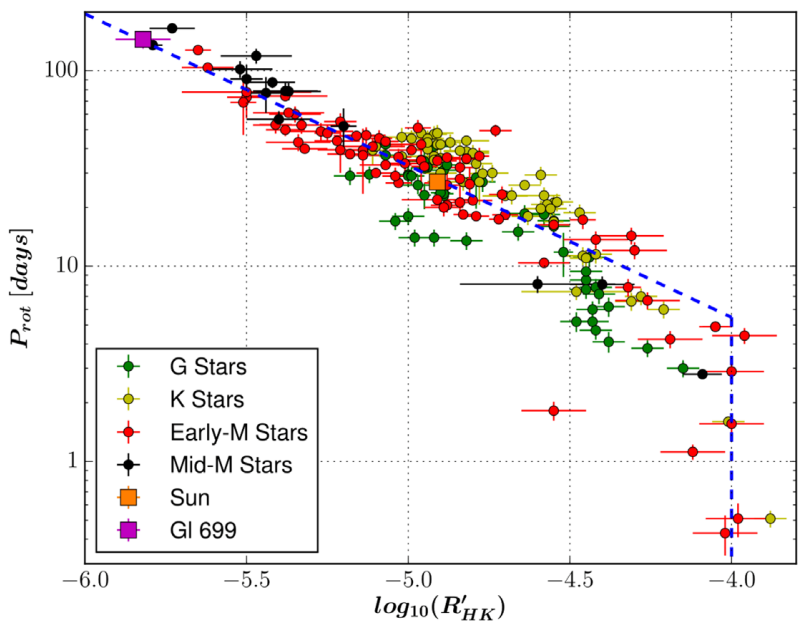

Figure 20. Rotation periodand chromospheric activity level $\log _{10} R_{\mathrm{HK}}^{\prime}$ of a G, K, Early-M (M0-M3), and Mid-M (M4-M6) stars sample from the literature, including the values obtained in this paper for G1 699. The blue line represents the relation obtained by Suárez Mascareño et al. (2018b) for M stars.

We computed the periodogram of the RV data using the Systemic console, including a linear trend term along with offset and jitter terms for each instrument as free parameters, which removes any possible long-term signal (of several years) that could be associated with either long-term activity or long-period planetary signals. The first periodogram after minimizing the linear term, offset, and jitter values provides the strongest signal at $233 \mathrm{~d}$ and secondary much less significant signals at 1 and $77 \mathrm{~d}$, but no significant signal around $145 \mathrm{~d}$. A real signal should appear at its original frequency and also at its two alias frequencies with a given significance depending on the level of noise (see e.g. Anglada-Escudé et al. 2013). The signal at $145 \mathrm{~d}$ does not appear in the periodogram of the RV data. However, we performed several tests with the published set of RV time series, trying to force a fit for the rotation signal (with both a sinusoid and a keplerian model), allowing the period to move in the range between 130 and $160 \mathrm{~d}$. After fitting and subtracting the stellar rotation signal, the $233 \mathrm{~d}$ signal remains highly significant in the periodogram of the residuals. We note that $77 \mathrm{~d}$ is about half of the estimated rotation period from activity indicators but we do not find any signal in the RV time series at about $145 \mathrm{~d}$. After fitting and subtracting the $77 \mathrm{~d}$ signal, the $233 \mathrm{~d}$ signal still holds with high power. The combined fit of the $77+233 \mathrm{~d}$ signals does not affect the final parameters of the planet reported in Ribas et al. (2018). We conclude that the planetary signal at $233 \mathrm{~d}$ is not related to any possible rotation signal present in the RV data, which we have not been able to detect since it is probably much weaker than the RV precision of the data. The RV analysis is extensively discussed in Ribas et al. (2018) in the context of the activity signal associated with stellar rotation and the planetary signal associated with Barnard b.

\section{CONCLUSIONS}

We have analysed the time variability of several spectroscopic indexes (H $\alpha$, Ca II H\&K, NaI D, and CCF's FWHM) in a sample of 964 spectra of Barnard's Star taken with seven different spectrographs (HARPS, HARPS-N, CARMENES, HIRES, APF, PFS, and UVES) in a time span of $14.5 \mathrm{yr}$. We also have used the available photometric time series of the star that forms a sample of 1390 measurements of photometric magnitudes coming from four different instruments (AAVSO, FCAPT-RCT, ASAS, and T90@SNO) in a time span of 15.1 yr.

We have detected the rotation period signal in the $\mathrm{H} \alpha$ and FWHM time series at 143 and $150 \mathrm{~d}$ respectively, along with a tentative detection of differential rotation between 130 and $180 \mathrm{~d}$ appearing also in the $\mathrm{NaD}$ time series. We determine the rotation period to be $145 \pm 15 \mathrm{~d}$ for Barnard's Star. We also calculate a chromospheric activity level of $\log _{10}\left(R_{\mathrm{HK}}^{\prime}\right)=-5.82$ that indicates a very low stellar activity. Using an activity-rotation relation, we obtain an expected rotation period that is in good agreement with our determination, and an upper limit to the activity induced RV signal associated with rotation of $1 \mathrm{~m} \mathrm{~s}^{-1}$. Also, the low X-ray activity of the star supports our determination of the stellar rotation period.

In the $\mathrm{CaHK}$ and $m_{\mathrm{V}}$ time series we find evidence of a long-term activity cycle in 3226 and $3846 \mathrm{~d}$, respectively, which is consistent with previous estimates of magnetic cycles from photometric time series in other M stars with similar activity levels. We then derive a long-term activity cycle of $3800 \pm 600 \mathrm{~d}$ for Barnard's Star.

We found no evidence that the signals detected in the chromospheric activity indicators are causing the RV signal detected by Ribas et al. (2018).

\section{ACKNOWLEDGEMENTS}

This work has been financed by the Spanish Ministry of Science, Innovation and Universities (MICIU) through the grant AYA201786389-P. BTP acknowledges Fundación La Caixa for the financial support received in the form of a Ph.D. contract. JIGH acknowledges financial support from the Spanish MICIU under the 2013 Ramón y Cajal program MICIU RYC-2013-14875. ASM acknowledges financial support from the Swiss National Science Foundation (SNSF). The IAA-CSIC and UCM teams acknowledge support by the Spanish Ministry of Economy and Competitiveness (MINECO) through grants AYA2016-79425-C31-P, AYA2016-79425-C3-2-P, AYA2016-79425-C3-3-P, ESP201454362P, and ESP2017-87143R. IR, JCM, MP, and EH acknowledge support from the Spanish MINECO and the Fondo Europeo de Desarrollo Regional (FEDER) through grant ESP2016-80435-C2$1-\mathrm{R}$, as well as the support of the Generalitat de Catalunya/CERCA program. GAE research is funded via the STFC Consolidated Grants ST/P000592/1, and a Perren foundation grant. The results of this paper were based on observations made with the Italian Telescopio Nazionale Galileo (TNG), operated on the island of La Palma by the INAF-Fundación Galileo Galilei at the Roque de Los Muchachos Observatory of the Instituto de Astrofísica de Canarias (IAC); observations made with the HARPS instrument on the ESO $3.6 \mathrm{~m}$ telescope at La Silla Observatory (Chile); observations made with the CARMENES instrument at the $3.5 \mathrm{~m}$ telescope of the Centro Astronómico Hispano-Alemán de Calar Alto (CAHA, Almería, Spain), funded by the German Max-Planck-Gesellschaft (MPG), the Spanish Consejo Superior de Investigaciones Científicas (CSIC), the European Union, and the CARMENES Consortium members. This paper made use of the IAC Supercomputing facility HTCondor (http://research.cs.wisc.edu/htcondor/), partly financed by the Ministry of Economy and Competitiveness with FEDER funds, code IACA13-3E-2493. We are grateful to all the observers of the projects whose data we are using for the following spectrographs: HARPS (072.C-0488, 183.C-0437, 191.C-0505, 099.C-0880), HARPSN (CAT14A_43, A27CAT_83, CAT13B_136, CAT16A_109, 
CAT17A_38, CAT17A_58), CARMENES (CARMENES GTO survey), HIRES $(\mathrm{U} 11 \mathrm{H}, \mathrm{U} 11 \mathrm{H}, \mathrm{N} 12 \mathrm{H}, \mathrm{N} 10 \mathrm{H}, \mathrm{A} 264 \mathrm{Hr}, \mathrm{A} 288 \mathrm{Hr}$, $\mathrm{C} 168 \mathrm{Hr}, \mathrm{C} 199 \mathrm{Hr}, \mathrm{C} 205 \mathrm{Hr}, \mathrm{C} 202 \mathrm{Hr}, \mathrm{C} 232 \mathrm{Hr}, \mathrm{C} 240 \mathrm{Hr}, \mathrm{C} 275 \mathrm{Hr}$, $\mathrm{C} 332 \mathrm{Hr}, \mathrm{H} 174 \mathrm{Hr}, \mathrm{H} 218 \mathrm{Hr}, \mathrm{H} 238 \mathrm{Hr}, \mathrm{H} 224 \mathrm{Hr}, \mathrm{H} 244 \mathrm{Hr}, \mathrm{H} 257 \mathrm{Hr}$, $\mathrm{K} 01 \mathrm{H}, \mathrm{N} 007 \mathrm{Hr}, \mathrm{N} 014 \mathrm{Hr}, \mathrm{N} 024, \mathrm{~N} 054 \mathrm{Hr}, \mathrm{N} 05 \mathrm{H}, \mathrm{N} 06 \mathrm{H}, \mathrm{N} 085 \mathrm{Hr}$, $\mathrm{N} 086 \mathrm{Hr}, \mathrm{N} 095 \mathrm{Hr}, \mathrm{N} 108 \mathrm{Hr}, \mathrm{N} 10 \mathrm{H}, \mathrm{N} 112 \mathrm{Hr}, \mathrm{N} 118 \mathrm{Hr}, \mathrm{N} 125 \mathrm{Hr}$, N129HR, N12H, N12H, N131Hr, N131Hr, N136Hr, N141Hr, $\mathrm{N} 145 \mathrm{Hr}, \mathrm{N} 148 \mathrm{Hr}, \mathrm{N} 14 \mathrm{H}, \mathrm{N} 157 \mathrm{Hr}, \mathrm{N} 15 \mathrm{H}, \mathrm{N} 168 \mathrm{Hr}, \mathrm{N} 19 \mathrm{H}, \mathrm{N} 20 \mathrm{H}$, $\mathrm{N} 22 \mathrm{H}, \mathrm{N} 28 \mathrm{H}, \mathrm{N} 32 \mathrm{H}, \mathrm{N} 50 \mathrm{H}, \mathrm{N} 59 \mathrm{H}, \mathrm{U} 014 \mathrm{Hr}, \mathrm{U} 01 \mathrm{H}, \mathrm{U} 023 \mathrm{Hr}$, $\mathrm{U} 027 \mathrm{Hr}, \mathrm{U} 027 \mathrm{Hr}, \mathrm{U} 030 \mathrm{Hr}, \mathrm{U} 052 \mathrm{Hr}, \mathrm{U} 058 \mathrm{Hr}, \mathrm{U} 05 \mathrm{H}, \mathrm{U} 064 \mathrm{Hr}$, U077Hr, U078Hr, U07H, U082Hr, U084Hr, U08H, U10H, U115Hr, $\mathrm{U} 11 \mathrm{H}, \mathrm{U} 12 \mathrm{H}, \mathrm{U} 131 \mathrm{Hr}, \mathrm{U} 142 \mathrm{Hr}, \mathrm{U} 66 \mathrm{H}, \mathrm{Y} 013 \mathrm{Hr}, \mathrm{Y} 065 \mathrm{Hr}, \mathrm{Y} 283 \mathrm{Hr}$, Y292Hr), UVES (65.L-0428, 66.C-0446, 267.C-5700, 68.C0415, 69.C-0722, 70.C-0044, 71.C-0498, 072.C0495, 173.C-0606, 078.C-0829), APF (LCES/APF planet survey), and PFS (CarnegieCalifornia survey).

\section{REFERENCES}

Adelman S. J., Boyd L., Dukes R. J., Jr, Guinan E. F., McCook G. M., Pyper D. M., 2001, in Batten A. H., ed., Proceedings of a Special Session of the XXIV General Assembly of the International Astronomical Union. Astronomy for delevoping countries. Astron. Soc. Pac. Manchester

Aigrain S. et al., 2015, MNRAS, 450, 3211

Alonso-Floriano F. J. et al., 2015, A\&A, 577, A128

Anglada-Escudé G. et al., 2013, A\&A, 556, A126

Anglada-Escudé G. et al., 2016, Nature, 536, 437

Astudillo-Defru N., Delfosse X., Bonfils X., Forveille T., Lovis C., Rameau J., 2017, A\&A, 600, A13

Barnard E. E., 1916, AJ, 29, 181

Benedict G. F. et al., 1998, AJ, 116, 429

Berdyugina S. V., Järvinen S. P., 2005, Astron. Nachr., 326, 283

Berta Z. K., Irwin J., Charbonneau D., Burke C. J., Falco E. E., 2012, AJ, 144,145

Boisse I., Bouchy F., Hébrard G., Bonfils X., Santos N., Vauclair S., 2011, A\&A, 528, A4

Brown A. G. A. et al., 2018, A\&A, 616, A1

Campbell B., Walker G. A. H., Yang S., 1988, ApJ, 331, 902

Chabrier G., Baraffe I., 2000, ARA\&A, 38, 337

Chabrier G., Küker M., 2006, A\&A, 446, 1027

Cosentino R. et al., 2012, The Messenger, 162, 9

Crane J. D., Shectman S. A., Butler R. P., Thompson I. B., Birk C., Jones P., Burley G. S., 2010 , in McLean I. S., Ramsay K., Takami H., eds, Proc. SPIE Conf. Ser. Vol. 7735, Ground-based and Airborne Instrumentation for Astronomy III. SPIE, Bellingham, p. 773553

Cumming A., 2004, MNRAS, 354, 1165

Dawson R. I., Fabrycky D. C., 2010, ApJ, 722, 937

Dekker H., D’Odorico S., Kaufer A., Delabre B., Kotzlowski H., 2000, in Iye M., Moorwood A. F. M., eds, Proc. SPIE Conf. Ser. Vol. 4008, Optical and IR Telescope Instrumentation and Detectors. SPIE, Bellingham, p. 534

Díaz R. F., Cincunegui C., Mauas P. J. D., 2007, MNRAS, 378, 1007

Díez-Alonso E. et al., 2019, A\&A, 621, A126

Dravins D., 1985, ASP Conf. Ser., IAU Colloq. 88: Stellar Radial Velocities, Astron. Soc. Pac., San Francisco, p. 311

Endl M., Kürster M., Els S., Hatzes A. P., Cochran W. D., 2001, A\&A, 374, 675

Gelderman R., 2001, ASP Conf. Ser. Vol. 246, Small Telescope Astronomy on Global Scales. Astron. Soc. Pac., San Francisco, p. 89

Gomes da Silva J., Santos N. C., Bonfils X., Delfosse X., Forveille T., Udry S., 2011, A\&A, 534, A30

Gomes da Silva J., Santos N. C., Bonfils X., Delfosse X., Forveille T., Udry S., Dumusque X., Lovis C., 2012, A\&A, 541, A9

Hartmann L., Soderblom D. R., Noyes R. W., Burnham N., Vaughan A. H., 1984, ApJ, 276, 254
Horne J. H., Baliunas S. L., 1986, ApJ, 302, 757

Hünsch M., Schmitt J. H. M. M., Sterzik M. F., Voges W., 1999, A\&AS, 135,319

Kiraga M., Stepien K., 2007, Acta Astron., 57, 149

Koen C., Kilkenny D., van Wyk F., Marang F., 2010, MNRAS, 403, 1949

Kürster M. et al., 2003, A\&A, 403, 1077

Lo Curto G. et al., 2015, The Messenger, 162, 9

Lomb N. R., 1976, Ap\&SS, 39, 447

Lovis C., Pepe F., 2007, A\&A, 468, 1115

Lovis C. et al., 2011, preprint (arXiv:1107.5325)

Marcy G. W., Butler R. P., Fischer D., Vogt S. S., Lissauer J. J., Rivera E. J., 2001, ApJ, 556, 296

Marino A., Micela G., Peres G., 2000, A\&A, 353, 177

Markwardt C. B., 2009, ASP Conf. Ser. Vol. 411, Astronomical Data Analysis Software and Systems XVIII. Astron. Soc. Pac., San Francisco, p. 251

Mayor M., Queloz D., 1995, Nature, 378, 355

Mayor M. et al., 2003, The Messenger, 114, 20

McQuillan A., Mazeh T., Aigrain S., 2014, ApJS, 211, 24

Meschiari S., Wolf A. S., Rivera E., Laughlin G., Vogt S., Butler P., 2009, PASP, 121, 1016

Newton E., Irwin J., Charbonneau D., Berta-Thompson Z. K., Dittmann J. A., West A. A., 2016a, ApJ, 821, 93

Newton E., Irwin J., Charbonneau D., Berta-Thompson Z. K., Dittmann J. A., 2016b, ApJ, 821, L19

Newton E. R., Mondrik N., Irwin J., Winters J. G., Charbonneau D., 2018, AJ, 156, 217

Noyes R. W., Hartmann L. W., Baliunas S. L., Duncan D. K., Vaughan A. H., 1984, ApJ, 279, 763

Passegger V. M. et al., 2018, A\&A, 615, A6

Paulson D. B., Allred J. C., Anderson R. B., Hawley S. L., Cochran W. D., Yelda S., 2006, PASP, 118, 227

Pavlenko Y., Suárez Mascareño A., Rebolo R., Lodieu N., Béjar V. J. S., González Hernández J. I., 2017, A\&A, 606, A49

Pepe F., Ehrenreich D., Meyer M. R., 2014, Nature, 513, 358

Pizzolato N., Maggio A., Micela G., Sciortino S., Ventura P., 2003, A\&A, 397, 147

Pojmański G., 1997, Acta Astron., 47, 467

Queloz D. et al., 2001, A\&A, 379, 279

Quirrenbach A. et al., 2018, in Evans C. J., Simard L., Takami H., eds, Proc. SPIE Conf. Ser. Vol. 10702, Ground-based and Airborne Instrumentation for Astronomy VII. SPIE, Bellingham, p. 107020W

Radick R. R., Lockwood G. W., Skiff B. A., Baliunas S. L., 1998, ApJS, 118,239

Reiners A., Schmitt J. H. M. M., 2003, A\&A, 398, 647

Reiners A., Schüssler M., Passegger V. M., 2014, ApJ, 794, 144

Reinhold T., Gizon L., 2015, A\&A, 583, A65

Reinhold T., Reiners A., Basri G., 2013, A\&A, 560, A4

Ribas I. et al., 2018, Nature, 563, 365

Robertson P., Endl M., Cochran W. D., Dodson-Robinson S. E., 2013, ApJ, 764, 3

Shappee P. et al., 2014, ApJ, 788, 48

Stumpff P., 1985, A\&A, 144, 232

Suárez Mascareño A., Rebolo R., González Hernández J. I., Esposito M., 2015, MNRAS, 452, 2745

Suárez Mascareño A., Rebolo R., González Hernández J. I., Esposito M., 2016, A\&A, 595, A12

Suárez Mascareño A., Rebolo R., González Hernández J. I., Esposito M., 2017, MNRAS, 468, 4772

Suárez Mascareño A. et al., 2018a, A\&A, 612, A41

Suárez Mascareño A. et al., 2018b, A\&A, 612, A89

Taylor J. R., 1982, An Introduction to Error Analysis. University Science Books, Mill Valley, CA

Vaiana G. S. et al., 1981, ApJ, 245, 163

Vanderburg A., Plavchan P., Johnson J. A., Ciardi D. R., Swift J., Kane S. R., 2016, MNRAS, 459, 3565 
Vaughan A. H., Preston G. W., Wilson O. C., 1978, PASP, 90, 267

Vogt S. S. et al., 1994, in D. L. Crawford, E. R. Craine, eds, Proc. SPIE Conf. Ser. Vol. 2198 , Instrumentation in Astronomy VIII. SPIE, Bellingham, p. 362

Vogt S. S. et al., 2014, PASP, 126, 359

Wargelin B. J., Saar S. H., Pojmański G., Drake J. J., Kashyap V. L., 2017, MNRAS, 464, 3281

Wildi F., Pepe F., Chazelas B., Lo Curto G., Lovis C., 2010, in McLean I. S., Ramsay S. K., Takami H., eds , Proc. SPIE Conf. Ser. Vol. 7735, Ground-based and Airborne Instrumentation for Astronomy III. SPIE, Bellingham, p. 77354X

Wolszczan A., Frail D. A., 1992, Nature, 355, 145

Wright N. J., Drake J. J., Mamajek E. E., Henry G. W., 2011, ApJ, 743,48

Wright N. J., Drake J. J., 2016, Nature, 535, 526

Zechmeister M., Kürster M., 2009, A\&A, 496, 577

Zechmeister M. et al., 2018, A\&A, 609, A12

\section{SUPPORTING INFORMATION}

Supplementary data are available at MNRAS online.

Table A1. Values of the three spectroscopic indexes and the FWHM with all the offsets applied, their respective errors, the BJD when the spectra was taken and the spectrograph used.

Table A2. Values of the photometric magnitudes with the offsets applied, their respective errors, the BJD when the measurement was taken and the instrument used.

Please note: Oxford University Press is not responsible for the content or functionality of any supporting materials supplied by the authors. Any queries (other than missing material) should be directed to the corresponding author for the article.

APPENDIX A: FULL DATA SET

Table A1. Values ofthe three spectroscopic indexes and the FWHM with all the offsets applied, their respective errors, the BJD when the spectra was taken and the spectrograph used.

\begin{tabular}{|c|c|c|c|c|c|c|c|c|c|}
\hline BJD-2450000 (d) & $\mathrm{H} \alpha$ & $\sigma \mathrm{H} \alpha$ & $\mathrm{CaHK}$ & $\sigma \mathrm{CaHK}$ & $\mathrm{NaD}$ & $\sigma \mathrm{NaD}$ & FWHM & $\sigma$ FWHM & Spectrograph \\
\hline 2763.3957 & 0.4959 & 0.0012 & & & & & & & UVES \\
\hline 2839.0807 & 0.5003 & 0.0016 & & & & & & & UVES \\
\hline 3082.3893 & 0.4763 & 0.0016 & & & & & & & UVES \\
\hline 3088.3837 & 0.5068 & 0.0017 & & & & & & & UVES \\
\hline 3099.3621 & 0.4815 & 0.0019 & & & & & & & UVES \\
\hline 3165.2780 & 0.4894 & 0.0013 & & & & & & & UVES \\
\hline 3191.1412 & 0.4725 & 0.0016 & & & & & & & UVES \\
\hline
\end{tabular}

Table A2. Values of thephotometricmagnitudes with the offsets applied, their respective errors, the BJD when the measurement was taken and the instrument used.

\begin{tabular}{lccc}
\hline BJD-2450000 (d) & $m_{\mathrm{V}}$ & $\sigma m_{\mathrm{V}}$ & Instrument \\
\hline 2524.5633 & 9.466392 & 0.011909 & ASAS \\
2535.5548 & 9.507276 & 0.010203 & ASAS \\
2544.5058 & 9.502228 & 0.010260 & ASAS \\
2547.5379 & 9.541082 & 0.009065 & ASAS \\
2549.5355 & 9.508656 & 0.008860 & ASAS \\
2552.5269 & 9.486937 & 0.011271 & ASAS \\
2558.4997 & 9.489218 & 0.011690 & ASAS \\
2697.9021 & 9.517063 & 0.009369 & ASAS \\
2701.8931 & 9.513012 & 0.008228 & ASAS \\
2711.8832 & 9.497800 & 0.009030 & ASAS
\end{tabular}


Table A3. AAVSO contributions.

\begin{tabular}{lccccc}
\hline Observer code & Name & Country & Filters & $N_{\text {exposures }}$ & $N_{\text {epochs }}$ \\
\hline BJFB & John Briol & USA & $V$ & 334 & 13 \\
BLOC & Lorenzo Barbieri & IT & $V, \mathrm{H} \alpha$ & 523,59 & $(9)$ \\
CIVA & Ivaldo Cervini & CH & $V, \mathrm{H} \alpha$ & 161,70 & $(12)$ \\
DLM & Marc Deldem & FR & $V$ & 2015 & 28 \\
DUBF & Franky Dubois & BE & $B V R I$ & $210,233,188,124$ & $(31)$ \\
HBB & Barbara Harris & USA & $V$ & 463 & 4 \\
HMB & Franz-Josef Hambsch & BE & $V$ & 2753 & 111 \\
KCLA & Clifford Kotnik & USA & $V, \mathrm{H} \alpha$ & 867,83 & $(8)$ \\
LJBE & Jean-Marie Lopez & FR & $V$ & 446 & 6 \\
MMAE & Michael McNeely & USA & $V$ & 2 & $(2)$ \\
OYE & Yenal Ogmen & CY & $V$ & 416 & 1 \\
PLFA & Luis Pérez & ES & $V$ & 65 & 1 \\
RZD & Diego Rodríguez & ES & $V$ & 1 & $(1)$ \\
SFGA & Fabián Sánchez Urquijo & EC & $V$ & 2 & $(2)$ \\
\hline
\end{tabular}

Note. Columns: Observer initials and name, country code (USA $=$ United States of America; IT = Italy; $\mathrm{CH}=$ Switzerland; FR = France; $\mathrm{BE}=$ Belgium; $\mathrm{CY}=$ Cyprus; $\mathrm{ES}=$ Spain; $\mathrm{EC}=$ Ecuador), filters, number of exposures, and number of epochs. The parenthesis in the last column indicates that the data sets were not included in the final analysis due to high scattering or insufficient number of observations.

${ }^{1}$ Instituto de Astrofísica de Canarias, E-38205 La Laguna, Tenerife, Spain ${ }^{2}$ Departamento de Astrofísica, Universidad de La Laguna, E-38206 La Laguna, Tenerife, Spain

${ }^{3}$ Instituto de Astrofísica de Andalucía, Glorieta de la Astronomía 1, E-18008 Granada, Spain

${ }^{4}$ Observatoire Astronomique de l'Université de Genève, CH-1290 Versoix, Switzerland

${ }^{5}$ Consejo Superior de Investigaciones Científicas, E-28006 Madrid, Spain

${ }^{6}$ Department of Terrestrial Magnetism, Carnegie Institution for Science, 5241 Broad Branch Road NW, Washington, DC 20015, USA

${ }^{7}$ Institut de Ciències de l'Espai, Campus UAB, C/Can Magrans s/n, E-08193 Bellaterra, Spain

${ }^{8}$ Institut d'Estudis Espacials de Catalunya (IEEC), E-08034 Barcelona, Spain

${ }^{9}$ School of Physics and Astronomy, Queen Mary University of London, 327 Mile End Rd, E14NS London, UK

${ }^{10}$ Institut für Astrophysik, Georg-August-Universität, Friedrich-Hund-Platz 1, D-37077 Göttingen, Germany

${ }^{11}$ Centro de Astrobiología, CSIC-INTA, ESAC campus, Camino bajo del castillo s/n, E-28692 Villanueva de la Cañada, Madrid, Spain

${ }^{12}$ Landessternwarte, Zentrum für Astronomie der Universität Heidelberg, Königstuhl 12, D-69117 Heidelberg, Germany

${ }^{13}$ UCO/Lick Observatory, University of California at Santa Cruz, 1156 High Street, Santa Cruz, CA 95064, USA

${ }^{14}$ The Observatories, Carnegie Institution for Science, 813 Santa Barbara Street, Pasadena, CA 91101, USA
${ }^{15}$ Departmento de Astronomía, Camino El Observatorio 1515, Universidad de Chile, Las Condes, Santiago, Chile

${ }^{16}$ Kavli Institute, Massachusetts Institute of Technology, 77 Massachusetts Avenue, Cambridge, MA 02139, USA

${ }^{17}$ Department of Exploitation and Exploration of Mines, University of Oviedo, E-33004 Oviedo, Spain

${ }^{18}$ Centre for Astrophysics Research, School of Physics, Astronomy and Mathematics, University of Hertfordshire, College Lane, AL10 9AB Hatfield, $U K$

${ }^{19}$ Warsaw University Observatory, Aleje Ujazdowskie 4, PL-00-478 Warszawa, Poland

${ }^{20}$ Department of Astrophysics and Planetary Science, Villanova University, Villanova, PA 19085, USA

${ }^{21}$ Centro Astronómico Hispano-Alemán (CSIC-MPG), Observatorio Astronómico de Calar Alto, Sierra de los Filabres, E-04550 Gérgal, Almería, Spain

${ }^{22}$ Departamento de Física de la Tierra y Astrofísica and IPARCOS-UCM (Instituto de Física de Partículas y del Cosmos de la UCM), Facultad CC. Físicas, Universidad Complutense de Madrid, E-28040, Madrid, Spain

${ }^{23}$ The American Association of Variable Star Observers, 49 Bay State Road, Cambridge, MA 02138, USA

${ }^{24}$ AstroLAB IRIS, Provinciaal Domein 'De Palingbeek', Verbrandemolenstraat 5, B-8902 Zillebeke, Ieper, Belgium

${ }^{25}$ Vereniging Voor Sterrenkunde (VVS), Oude Bleken 12, 2400 Mol, Belgium

This paper has been typeset from a $\mathrm{T}_{\mathrm{E}} \mathrm{X} / \mathrm{ET} \mathrm{E} \mathrm{X}$ file prepared by the author. 